\title{
APPLICATIONS OF GRAVITATIONAL LENSING IN COSMOLOGY
}

\author{
MATTHIAS BARTELMANN \\ Zentrum für Astronomie, ItA, Universität Heidelberg, Albert-Überle-Str. 2, 69120 Heidelberg, Germany
}

\begin{abstract}
Gravitational lensing originates from the deflection of light by masses, irrespective of their physical state or composition. Since it appears inescapable that most of the matter in the universe is dark, gravitational lensing has developed into one of the primary tools to learn about the amount, composition and distribution of masses in the universe.

The review will summarise the theory of gravitational lensing, starting from Fermat's principle. This will first be applied to isolated lenses like compact objects, galaxies, and galaxy clusters. Cosmologically relevant applications will be described, such as searches for compact dark-matter objects in galactic halos, measurements of the Hubble constant in galaxy lenses, and methods for mapping the dark matter in galaxy clusters. Next, the theory of cosmological lensing will be introduced. The concepts of lensing by large-scale structures and its measurement will be discussed, concluding with an overview of results which have so far been obtained, and an outlook at what can be expected in the near future.
\end{abstract}

\section{INTRODUCTION}

\subsection{The dark Universe}

It seems that we have to accept that most matter in the Universe is dark, and that the majority of the cosmic energy density is not contributed by matter. These conclusions appear as the most straightforward interpretation of numerous cosmological measurements within the class of Friedmann-Lemaittre cosmological models.

Those models are derived from Einstein's general theory of relativity using the two symmetry assumptions that the Universe is isotropic around us, and that our position in the Universe is by no means preferred to others. Together, these two assumptions imply that the Universe is isotropic around any of its points, and thus homogeneous.

The assumption that the Universe is isotropic around us seems dubious at first sight, but is very well satisfied if the observable Universe is averaged over sufficiently large scales which are still small compared to its overall size. Assuming our cosmic position not to be preferred compared to any other is a generalisation of the Copernican principle, which said that the Earth is not at the centre of the Solar System.

Given these two symmetry assumptions, Einstein's generally very complicated field equations reduce to two simple, ordinary differential equations for the scale factor of the Universe, the socalled Friedmann equations. They admit families of solutions characterised by a set of parameters like the densities of various energy contributions, the so-called cosmological constant, the current expansion rate of the Universe or Hubble constant, and so forth. Determining the global properties of the Universe is then reduced to measuring these parameters, which used to be the formidable paramount task of modern cosmology.

The past few years have seen a breakthrough. Mainly three types of cosmological measurement have allowed virtually all relevant cosmological parameters to be precisely constrained. Among the most interesting and puzzling of their findings are that the total density of all forms of energy together sums up to a critical value which makes space flat. Radiation contributes negligibly today to this critical density, matter has just about 30 per cent, and the rest is some different, mysterious form of energy. Of the matter, only about 15 per cent is of the "ordinary", baryonic form that we know from Earth, which consists of pro- tons, neutrons and electrons. The remainder of the matter cannot interact with light and is thus called dark matter .

We are thus put into the embarrassing situation of now knowing quite precisely how much energy per volume there is in the Universe, but having no precise idea what the vast majority of this energy may be composed of. Einstein's theory of special relativity has taught us that matter and energy are equivalent. Yet, is does make sense to distinguish dark matter from dark energy : Dark matter can clump and form structures, while dark energy cannot and is thus smoothly distributed.

A possible form of the dark energy could be the cosmological constant which Einstein introduced into his equations when Friedmann found that generally-relativistic cosmological models are unstable and either expand or contract. At that time towards the end of the 1920s, Einstein believed the Universe to be static, which he could achieve only if he included a repulsive term into his equations, which could prevent the Universe from collapsing under its own gravity. This term, which is proportional to the cosmological constant, was soon considered obsolete when Slipher and Hubble found that the Universe is not static but expanding (Hubble 1929; Hubble \& Humason 1931). However, evidence is mounting that the cosmological constant, or something behaving similarly, is in fact required.

In this situation, which the recent advances of observational cosmology have made quite inescapable, two of the most pressing cosmological questions are, "What is the dark matter composed of, and how is it distributed?", and "What is the dark energy, and how does it evolve in time?" With both those dominant constituents of the Universe not interacting with light, the investigation of these two questions requires methods which reveal structures even if they do not emit or absorb electromagnetic radiation.

\subsection{The role of gravitational lensing}

Another effect following from Einstein's theory of general relativity comes to assist. Masses bend light paths such that they appear curved towards those, very much like light rays passing through convex glass lenses. This effect immediately follows from the equivalence principle, which says that gravitational forces cannot be distinguished from inertial forces experienced in an accelerating frame of reference. This was the heuristic guiding principle for Einstein's construction of general relativ- 
ity, and it thus became clear very early during the development of the theory that gravitational light deflection would be one of its consequences. It was a triumph for Einstein when the light deflection by the Sun was discovered in 1919 (Eddington 1919).

For deflecting light, matter (or energy) needs to be inhomogeneously distributed. Perfectly homogeneous matter is thus invisible even for gravitational lensing . However, lensing can also be employed for studying the dark energy, even though it is (or can be) defined as a perfectly smooth background of energy.

If the dark energy is well described as a cosmological constant, it does not evolve in time. However, the cosmological constant is unsatisfactory from the point of view of theoretical physics because its density deviates by 120 orders of magnitude from expectations derived from particle physics. Other models for the dark energy typically predict it to change with time. Thus, it is in fact a question of fundamental physical importance how the dark energy is evolving as the Universe expands. This becomes measurable because the dark energy changes the expansion behaviour of the Universe and thereby the growth of structures in it. Depending on the time evolution of the dark energy, cosmic structures form earlier or later in cosmic history. Thus, using gravitational lensing for investigating matter inhomogeneities in dependence of their cosmic age gives indirect clues as to the nature of the dark energy, even though it does not form structures.

\section{BASIC PRINCIPLES OF GRAVITATIONAL LENSING}

This section briefly summarises the foundation of gravitational lensing theory. Readers not interested in the mathematics can immediately skip to subsection 2.4 where the basic physical properties of lensing will be summarised.

\subsection{Fermat's principle and the deflection angle}

The theory of gravitational lensing is most easily built up starting from Fermat's principle, which is well known from geometrical optics. It holds that between a fixed source and a fixed observer, light will choose a path along which its travel time is extremal. Wave optics is typically unimportant for gravitational lensing because the wave length of light is incommensurably smaller than any structures in a gravitational lens 1

The light travel time is calculated in geometrical optics using the index of refraction $n$, which quantifies by how much the light speed is lower in a medium compared to vacuum. In general relativity, the light speed is reduced in presence of a gravitational field.

For by far the most astrophysical applications of gravitational lensing, the gravitational field can be described by a small Newtonian gravitational potential $\Phi \ll c^{2}$ which moves with velocities small compared to the speed of light. Under these conditions, the index of refraction is

$$
n=1-\frac{2 \Phi}{c^{2}}
$$

from which Fermat's principle yields the deflection angle

$$
\hat{\vec{\alpha}}=\frac{2}{c^{2}} \int \mathrm{d} l \vec{\nabla}_{\perp} \Phi
$$

where $\vec{\nabla}_{\perp}$ is the gradient perpendicular to the light ray along which the integral is to be carried out. However, typical deflection angles being very small, is is permissible to evaluate the

\footnotetext{
${ }^{1}$ Wave optics can be relevant, however, for gravitational lensing of gravitational waves.
}

integral along the unperturbed, straight light path. This corresponds to Born's approximation familiar from scattering theory.

A point mass $M$ has the gravitational potential

$$
\Phi=-\frac{G M}{r}
$$

where $G$ is the gravitational constant and $r$ is the distance from the mass. Arranging the coordinate system such that the unperturbed light path propagates into the positive $z$ direction, we find

$$
\hat{\vec{\alpha}}=\frac{4 G M}{c^{2}} \frac{\vec{b}}{b^{2}},
$$

where $\vec{b}$ is the vector connecting the point mass and the light ray in the $x-y$ plane perpendicular to the light ray containing the mass. This plane is called the lens plane . Lensing by an isolated lens is reduced to a sharp kink in the lens plane of the otherwise straight light path.

The deflection angle is directed towards the lens and proportional to the mass and the inverse separation $b$ between lens and light ray. Since $2 G M / c^{2}$ is the Schwarzschild radius $R_{\mathrm{S}}$ of the point mass, we can write

$$
\hat{\alpha}=\frac{2 R_{\mathrm{s}}}{b}
$$

The linear dependence of the deflection angle on the lens mass allows it to be easily generalised for extended mass distributions. Projecting the lensing mass distribution into the lens plane gives the surface mass density

$$
\Sigma(\vec{b})=\int \mathrm{d} z \rho(\vec{b}, z)
$$

from the three-dimensional mass density $\rho$. The projected lens is then decomposed into infinitesimal mass elements $\Sigma\left(\vec{b}^{\prime}\right) \mathrm{d}^{2} b^{\prime}$ which can individually be considered as point masses. The total deflection angle at a point $\vec{b}$ is then the sum of point-mass deflection angles,

$$
\hat{\vec{\alpha}}(\vec{b})=\frac{4 G}{c^{2}} \int \mathrm{d}^{2} b^{\prime} \frac{\Sigma\left(\vec{b}^{\prime}\right)\left(\vec{b}-\vec{b}^{\prime}\right)}{\left|\vec{b}-\vec{b}^{\prime}\right|^{2}} .
$$

It is convenient to use angular coordinates $\theta_{1,2}$ on the lens plane instead of the physical coordinates $\vec{b}$. Introducing the distance $D_{1}$ of the lens from the observer, $\vec{b}=D_{1} \vec{\theta}$ and

$$
\hat{\vec{\alpha}}(\vec{\theta})=\frac{4 G}{c^{2}} D_{1} \int \mathrm{d}^{2} \theta^{\prime} \frac{\Sigma\left(D_{1} \vec{\theta}^{\prime}\right)\left(\vec{\theta}-\vec{\theta}^{\prime}\right)}{\left|\vec{\theta}-\vec{\theta}^{\prime}\right|^{2}} .
$$

\subsection{Lens equation and lensing potential}

We now introduce a source plane parallel to the lens plane at distance $D_{\mathrm{s}}$ from the observer, and cover it with angular coordinates $\beta_{1,2}$. If the coordinate origin is suitably chosen, coordinates $\vec{\beta}$ and $\vec{\theta}$ are related by

$$
D_{\mathrm{s}} \vec{\beta}=D_{\mathrm{s}} \vec{\theta}-D_{\mathrm{ls}} \hat{\vec{\alpha}}(\vec{\theta}),
$$

where $D_{\mathrm{ls}}$ is the distance between lens and source. Introducing the reduced deflection angle

$$
\vec{\alpha}(\vec{\theta}):=\frac{D_{\mathrm{ls}}}{D_{\mathrm{s}}} \hat{\vec{\alpha}}(\vec{\theta})=\frac{4 G}{c^{2}} \frac{D_{\mathrm{l}} D_{\mathrm{ls}}}{D_{\mathrm{s}}} \int \mathrm{d}^{2} \theta^{\prime} \frac{\Sigma\left(D_{1} \vec{\theta}^{\prime}\right)\left(\vec{\theta}-\vec{\theta}^{\prime}\right)}{\left|\vec{\theta}-\vec{\theta}^{\prime}\right|^{2}},
$$


the lens equation simply becomes

$$
\vec{\beta}=\vec{\theta}-\vec{\alpha}(\vec{\theta})
$$

It is the non-linearity of this apparently simple equation due to the dependence of $\vec{\alpha}$ on $\vec{\theta}$ which makes it physically interesting. Among other things, it implies that gravitational lenses can produce multiple images of single sources when the inversion of the lens equation, i.e. the determination of image positions $\vec{\theta}$ from source positions $\vec{\beta}$, is no longer unique.

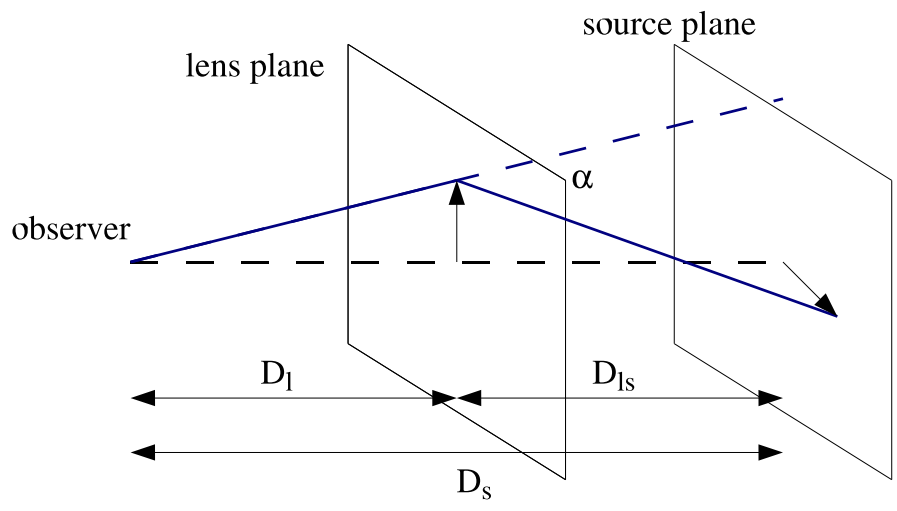

FIG. 1.-Sketch illustrating the geometry of imaging by an isolated lens

Following (4), the reduced deflection angle of a point mass is

$$
\alpha(\theta)=\frac{4 G M}{c^{2} \theta} \frac{D_{\mathrm{ls}}}{D_{\mathrm{l}} D_{\mathrm{s}}} .
$$

A source exactly behind the lens, i.e. at $\vec{\beta}=0$, is imaged according to (11) as a ring with radius

$$
\theta_{\mathrm{E}}=\left[\frac{4 G M}{c^{2}} \frac{D_{\mathrm{ls}}}{D_{\mathrm{l}} D_{\mathrm{s}}}\right]^{1 / 2}=\left[\frac{2 R_{\mathrm{s}} D_{\mathrm{ls}}}{D_{\mathrm{l}} D_{\mathrm{s}}}\right]^{1 / 2},
$$

which is called the Einstein radius. It sets a typical scale for the separation of multiple images. Assuming $2 D_{\mathrm{ls}} \simeq D_{\mathrm{s}}$ in typical situations, (13) shows that the Einstein radius is typically given by the square root of the ratio between the Schwarzschild radius of the lens and its distance to the observer, $\left(R_{\mathrm{S}} / D_{1}\right)^{1 / 2}$. The Schwarzschild radius of a star is of order $10^{5} \mathrm{~cm}$. If it is within our Galaxy, $D_{1} \simeq 3 \times 10^{22} \mathrm{~cm}$, thus $\theta_{\mathrm{E}} \simeq 2 \times 10^{-9}$ radians, or $4 \simeq 10^{-4}$ arc seconds. For a galaxy at cosmological distances, $R_{\mathrm{S}} \simeq 10^{16} \mathrm{~cm}$ and $D_{\mathrm{l}} \simeq 10^{27} \mathrm{~cm}$, thus $\theta_{\mathrm{E}} \simeq 3 \times 10^{-6}$ radians or $\simeq 0.6$ arc seconds. Galaxy clusters at cosmological distances have Einstein radii of order 50 larger than that of galaxies, or $\simeq 30$ arc seconds.

The surface-mass density $\Sigma$ becomes dimension-less when divided by the critical surface mass density

$$
\Sigma_{\mathrm{cr}}:=\frac{c^{2}}{4 \pi G} \frac{D_{\mathrm{s}}}{D_{\mathrm{l}} D_{\mathrm{ls}}}
$$

Introducing the convergence $\kappa(\vec{\theta})$ as the ratio $\Sigma\left(D_{1} \vec{\theta}\right) / \Sigma_{\mathrm{cr}}$, the reduced deflection angle reads

$$
\vec{\alpha}(\vec{\theta})=\frac{1}{\pi} \int \mathrm{d}^{2} \theta^{\prime} \frac{\kappa\left(\vec{\theta}^{\prime}\right)\left(\vec{\theta}-\vec{\theta}^{\prime}\right)}{\left|\vec{\theta}-\vec{\theta}^{\prime}\right|^{2}}
$$

Poisson's equation relates the three-dimensional density $\rho$ to the gravitational potential,

$$
\vec{\nabla}^{2} \Phi=4 \pi G \rho .
$$

The projection (6) implies the Poisson equation for the convergence $\kappa$,

$$
\frac{\Sigma(\vec{\theta})}{\Sigma_{\mathrm{cr}}}=\kappa(\vec{\theta})=\frac{1}{2} \vec{\nabla}^{2} \psi(\vec{\theta})
$$

with the lensing potential

$$
\psi(\vec{\theta})=\frac{2}{c^{2}} \frac{D_{\mathrm{ls}}}{D_{\mathrm{l}} D_{\mathrm{s}}} \int \mathrm{d} z \Phi\left(D_{\mathrm{l}} \vec{\theta}, z\right)
$$

Using the Green's function of the two-dimensional Laplacian operator, Eq. 17 can be immediately be solved for $\psi(\vec{\theta})$,

$$
\psi(\vec{\theta})=\frac{1}{\pi} \int \mathrm{d}^{2} \theta^{\prime} \kappa\left(\vec{\theta}^{\prime}\right) \ln \left|\vec{\theta}-\vec{\theta}^{\prime}\right|
$$

A comparison with 15 shows that the (reduced) deflection angle is the gradient of the (reduced) lensing potential,

$$
\vec{\alpha}(\vec{\theta})=\vec{\nabla} \psi(\vec{\theta}) .
$$

As in geometrical optics, light deflection is described as a consequence of reduced light speed, either in a medium like glass or in a gravitational field. The index of refraction introduced above quantifies the actual light speed $c^{\prime}$ in terms of the light speed $c$ in vacuum, $c^{\prime}=c / n$. The reduced speed leads to an enhanced travel time (Shapiro delay) in a gravitational field,

$$
t^{\prime}=\int \frac{\mathrm{d} z}{c^{\prime}}=\frac{1}{c} \int \mathrm{d} z n=\frac{1}{c} \int \mathrm{d} z\left(1-\frac{2 \Phi}{c^{2}}\right)=t-\frac{2}{c^{3}} \int \mathrm{d} z \Phi,
$$

where $t$ is the travel time in vacuum.

Due to the light deflection, the light path in presence of a gravitational field is also geometrically longer. Thus, gravitational lenses cause a two-fold time delay which is composed of a gravitational and a geometrical contribution. Travel times from the source to the observer along different paths belonging to multiple images are thus typically different. This time delay becomes observable with variable sources. Features in their light curves such as sudden rises or drops appear first in the image with the shortest travel time, and then repeat with a certain delay in the other images.

These relations were obtained assuming an isolated lens, i.e. a lensing mass distribution which is small compared to the cosmological distances which typically separate sources, lenses and the observer. When lensing by mass distributions on cosmological scales needs to be taken into account, the mathematical description complicates considerably. However, the end result is that an effective lensing potential

$$
\psi(\vec{\theta})=\frac{2}{c^{2}} \int_{0}^{w} \mathrm{~d} w^{\prime} \frac{D\left(w-w^{\prime}\right)}{D(w) D\left(w^{\prime}\right)} \Phi\left[D\left(w^{\prime}\right) \vec{\theta}, w^{\prime}\right]
$$

can be introduced such that the total deflection angle experienced by a light ray is again $\vec{\nabla} \psi$, and an effective convergence is given by Poisson's equation (16). In (22), $w$ and $D(w)$ are the comoving coordinate and angular diameter distances in the possibly curved background Universe. If space is flat, $w=D(w)$. The sources are assumed at distance $w$, and the integration includes all lenses at lower distances $w^{\prime}$. The similarity to 18 is evident.

These are not merely formal developments. The fact that the lensing deflection angle is the gradient of a scalar potential has profound implications for the astrophysical applications of gravitational lensing, as we shall see shortly. 


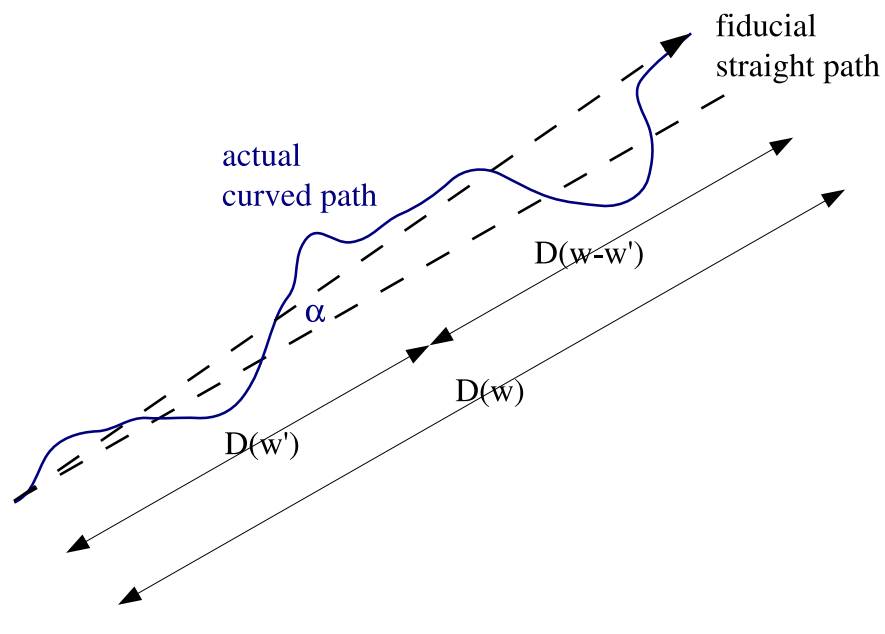

FIG. 2.- Illustration of lensing by large-scale structures

\subsection{Local imaging properties}

Typical sources are small compared to the lenses. Imaging by gravitational lensing can then be described by the linearised lens equation. Let the centre of the source be at $\vec{\beta}_{0}$ and its image at $\vec{\theta}_{0}$, where the deflection angle be $\vec{\alpha}_{0}:=\vec{\alpha}\left(\vec{\theta}_{0}\right)$. Then, the deflection angle at a neighbouring image point $\vec{\theta}=\vec{\theta}_{0}+\delta \vec{\theta}$ is, to first approximation,

$$
\vec{\alpha}=\vec{\alpha}_{0}+\left.\frac{\partial \vec{\alpha}}{\partial \vec{\theta}}\right|_{\vec{\theta}_{0}} \delta \vec{\theta},
$$

and the lens equation simplifies to

$$
\delta \vec{\beta}=\vec{\beta}-\vec{\beta}_{0}=\delta \vec{\theta}-\left.\frac{\partial \vec{\alpha}}{\partial \vec{\theta}}\right|_{\vec{\theta}_{0}} \delta \vec{\theta}=\mathscr{A} \delta \vec{\theta}
$$

where $\mathscr{A}$ is the Jacobian matrix of the lens equation with components

$$
\mathscr{A}_{i j}=\frac{\partial \beta_{i}}{\partial \theta_{j}}=\delta_{i j}-\frac{\partial \alpha_{i}}{\partial \theta_{j}}=\delta_{i j}-\frac{\partial^{2} \psi}{\partial \theta_{i} \partial \theta_{j}},
$$

where we have used that $\vec{\alpha}$ is the gradient of $\psi$.

The matrix $\mathscr{A}$ is evidently symmetric. Its trace is related to the convergence $\kappa$ by

$$
\operatorname{tr} \mathscr{A}=\mathscr{A}_{11}+\mathscr{A}_{22}=2(1-\kappa) .
$$

Accordingly, the matrix $\mathscr{A}$ can be decomposed as

$$
\mathscr{A}=\left(\begin{array}{cc}
1-\kappa-\gamma_{1} & -\gamma_{2} \\
-\gamma_{2} & 1-\kappa+\gamma_{1}
\end{array}\right),
$$

with the shear components

$$
\gamma_{1}:=\frac{1}{2}\left(\frac{\partial^{2} \psi}{\partial \theta_{1}^{2}}-\frac{\partial^{2} \psi}{\partial \theta_{2}^{2}}\right), \quad \gamma_{2}:=\frac{\partial^{2} \psi}{\partial \theta_{1} \partial \theta_{2}},
$$

which form the trace-free, symmetric shear matrix.

If there is no shear, $\gamma_{1,2}=0$, the matrix $\mathscr{A}$ becomes proportional to the unit matrix. Then, lensed sources appear isotropically stretched or shrunk, but undistorted. Conversely, the shear $\left(\gamma_{1}, \gamma_{2}\right)$ is responsible for image distortions.
Typically, the images will cover a different solid angle than the source. This is quantified by the determinant of $\mathscr{A}$, whose inverse is the magnification factor,

$$
\mu=\frac{1}{\operatorname{det} \mathscr{A}}=\frac{1}{(1-\kappa)^{2}-\gamma_{1}^{2}-\gamma_{2}^{2}} .
$$

The magnification will become very large where det $\mathscr{A} \rightarrow 0$. Points $\vec{\theta}_{\mathrm{c}}$ in the lens plane where det $\mathscr{A}=0$ are called critical points. They form closed, so-called critical curves. Their images in the source plane,

$$
\vec{\beta}_{\mathrm{c}}=\vec{\theta}_{\mathrm{c}}-\vec{\alpha}\left(\vec{\theta}_{\mathrm{c}}\right),
$$

are called caustic curves, or caustics. A source close to a caustic has highly magnified images next to a critical curve.

Apart from identifying regions of highest magnification in the source or lens planes, critical curves and caustics also separate areas of different image multiplicity. Far away from a lens, a single source must have a single image. When the source crosses a caustic on its way towards the (projected) lens centre, its image number increases by two. These additional images appear next to the critical curve belonging to the caustic. As a corollary, this implies that odd numbers of images are expected from gravitational lenses. The fact that even image numbers are typically observed allows interesting conclusions on the lensing mass distributions.

\subsection{Basic properties of gravitational lensing}

Summarising the preceding subsections, we see that gravitational lensing leads to a variety of phenomena which can qualitatively be understood in an intuitive way. First, the reduced light speed in a gravitational field causes light to be deflected much as in ordinary geometrical optics. This gravitational light deflection is differential, which means that neighbouring light rays experience slightly different deflections. Consequently, images typically appear enlarged or shrunk and distorted compared to the sources. These imaging properties are described by the convergence $\kappa$, which is proportional to the surface mass density of the lens and responsible for isotropic stretching or shrinking of images, and the two-component shear $\gamma_{1,2}$, which is responsible for image distortions. Together, convergence and shear cause the image magnification, i.e. the change of the total solid angle covered by an image compared to source.

Due to their deflection, light rays from a single source can reach the observer along multiple paths, leading to multiple images. Gravitational time delay and the geometrically longer deflected light paths cause a net time delay which is generally different for each of the images, leading to time delays between multiple images of a single source.

All properties of gravitational lensing can be summarised by a scalar potential $\psi$ whose gradient is the deflection angle. This statement holds true for individual, localised lenses which are small compared to the distance from the source to the observer such as stars, galaxies or galaxy clusters, but also for extended lenses of cosmological scale such as the large-scale structure of the Universe.

\subsection{Simple lens models}

Important mass distributions often used for understanding basic lensing properties of astrophysical objects, or for modelling image configurations of observed lenses, are the point mass, the isothermal sphere, and the (generalised) Navarro-Frenk-White (NFW) profile. They are intrinsically axially symmetric, but can easily be distorted to mimic asymmetric mass distributions. 

is

The point mass was already introduced. Its lensing potential

$$
\psi(\theta)=\frac{4 G M}{c^{2}} \frac{D_{\mathrm{ds}}}{D_{\mathrm{d}} D_{\mathrm{s}}} \ln |\theta| .
$$

Point masses are an essential tool especially for microlensing studies.

The isothermal sphere has a three-dimensional density profile falling as $r^{-2}$. It may be singular or have a core with finite density within a core radius $r_{\mathrm{c}}$. Its lensing potential is

$$
\psi(\theta)=\frac{4 \pi \sigma^{2}}{c^{2}} \frac{D_{\mathrm{ds}}}{D_{\mathrm{s}}} \sqrt{\theta_{1}^{2}+\theta_{2}^{2}+\theta_{\mathrm{c}}^{2}},
$$

where the angular core radius $\theta_{\mathrm{c}}=r_{\mathrm{c}} / D_{1}$ may vanish. Since circular velocities in isothermal spheres do not depend on radius, they naturally reproduce flat rotation curves, which is why they are often used for modelling galaxy lenses.

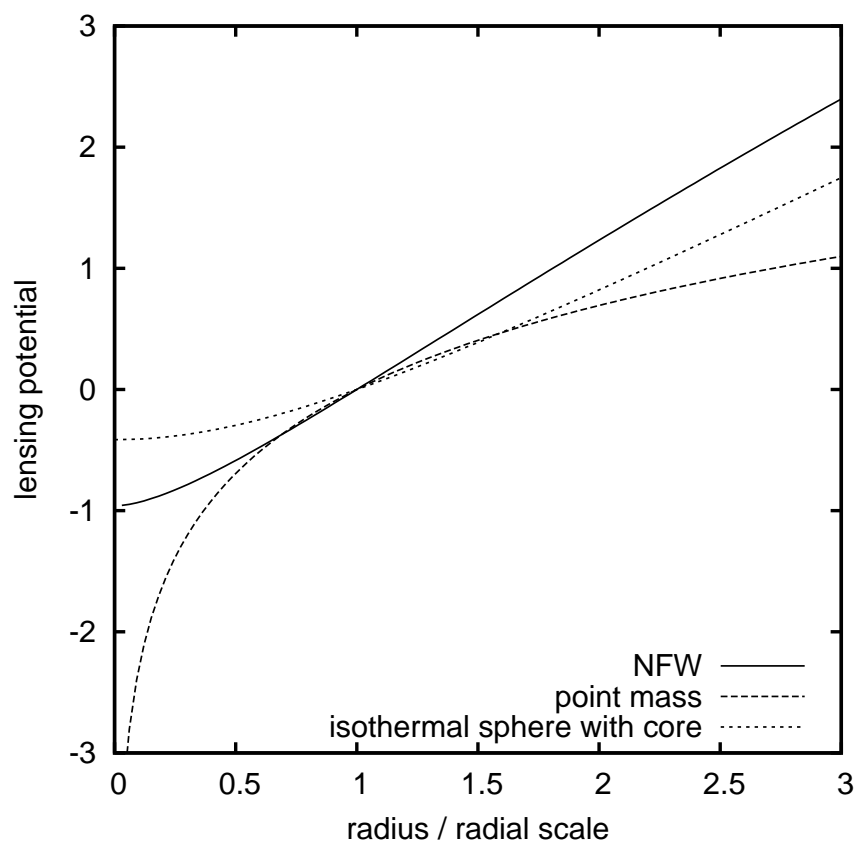

FIG. 3.- Radial dependence of three different lensing potentials: the NFW profile (solid line), the point mass (dashed curve), and the nonsingular isothermal sphere (dotted curve). All curves are arbitrarily normalised such as to pass through zero at a dimension-less radius of unity.

Numerical simulations showed that dark-matter halos consistently have density singular profiles which are shallow inside and steep outside a scale radius $r_{\mathrm{s}}$. Generally,

$$
\rho(r)=\frac{\rho_{\mathrm{s}}}{x^{\alpha}(1+x)^{3-\alpha}}
$$

with $x:=r / r_{\mathrm{s}}$. In its original form (suggested by Navarro et al. 1996, 1997), $\alpha=1$. Other simulations found steeper central slopes, $\alpha \simeq 1.5$. More recent simulations show that $\alpha$ does not converge to a fixed value down to the smallest resolved scales, but rather gently approaches unity towards the halo centres. The lensing potential of the NFW profile $(\alpha=1)$ reads

$$
\psi(\theta)=4 \kappa_{\mathrm{s}}\left[\frac{1}{2} \ln ^{2} \frac{x}{2}-2 \operatorname{arctanh}^{2} \sqrt{\frac{1-x}{1+x}}\right],
$$

with $\kappa_{\mathrm{s}}:=\rho_{\mathrm{s}} r_{\mathrm{s}} \Sigma_{\mathrm{cr}}^{-1}$ (Meneghetti et al. 2003b).
Deviations from axial symmetry are generally necessary to explain observed image configurations. The simplest approach is to replace the radius $\theta$ by

$$
\theta^{\prime}:=\left[(1-\varepsilon) \theta_{1}^{2}+\frac{\theta_{2}^{2}}{1-\varepsilon}\right]^{1 / 2}
$$

in the lensing potential. This elliptically deformed lensing potential has the disadvantage that its Laplacian is dumbbellshaped and not non-negative for moderate and large ellipticities $\varepsilon$, which implies an unphysical surface-mass density. Similar elliptical deformations of the convergence $\kappa$ are thus preferred, albeit much more difficult to study.

It is often necessary to embed lenses into an environment which supplies constant shear, and possibly also constant convergence. Examples are stars in galaxies, or galaxies in galaxy clusters. In both cases, the lenses are small compared to the scale on which the properties of their surroundings change. Constant convergence $\kappa_{0}$ and constant shear components $\gamma_{01,2}$ can be described by the effective lensing potential

$$
\psi_{0}(\theta)=\left(\kappa_{0}+\gamma_{01}\right) \theta_{1}^{2}+\left(\kappa_{0}-\gamma_{01}\right) \theta_{2}^{2}+\gamma_{02} \theta_{1} \theta_{2}
$$

which may be added to the lensing potential of an individual lens if needed.

\section{STRUCTURE AND CONTENTS OF GALAXIES}

\subsection{The Galaxy}

We do not know what the dark matter consists of. We know that it must not interact electromagnetically, because otherwise the cosmic microwave background would show temperature fluctuations on the level of $10^{-3} \mathrm{~K}$ rather than $10^{-5} \mathrm{~K}$. We also know that the dark matter must be cold in the sense that the velocity of its constituents must be small compared to the speed of light, because otherwise the large-scale distribution of the galaxies would be different. It is likely that this cold dark matter is composed of weakly-interacting elementary particles, but it could equally well consist of compact objects like, e.g. low-mass black holes.

Gravitational lensing provides one way to test this possibility. Our galaxy, the Milky Way, is expected to be embedded into a halo which predominantly consists of dark matter. If that dark matter was composed of compact objects rather than elementary particles, lines-of-sight out of the Galaxy would occasionally pass nearby one of those. They would act as point-mass lenses on sources in their background. Although their image splitting would be substantially below the detection threshold, they would cause a well-measurable magnification (Paczyński 1986).

Quite independent on the mass spectrum of these hypothetical compact objects (called MACHO 2 ), the probability of any one of them causing a microlensing event at any instant in time is of order $(v / c)^{2}$, where $v \simeq 220 \mathrm{~km} \mathrm{~s}^{-1} \mathrm{Mpc}^{-1}$ is a typical velocity for the stars in the Galaxy. Consequently, this microlensing optical depth is of order $10^{-6}$. Finding its magnification signature thus requires of order $10^{6}$ light curves to be monitored.

Originally perceived more like science fiction, projects were carried out which observed sufficient numbers of stars in the Large and Small Magellanic Clouds (LMC and SMC, respectively) with sufficient accuracy and time sampling for detecting some microlensing events among the overwhelming signal from variable stars.

\footnotetext{
${ }^{2}$ acronym for massive compact halo objects
} 
Analysing data taken from 11.9 million stars over 5.7 years, the MACHO project (Alcock et al. 2000) found 13-17 events, while between 2 and 4 were expected from known stellar populations in the Milky Way and the LMC. The microlensing optical depth deduced from lensing events lasting between 2 and 400 days is $\tau=1.2_{-0.3}^{+0.4} \times 10^{-7}$. This implies that between $8 \%$ and $50 \%$ of the Milky Way's halo can be composed of MACHOs (at 95\% confidence), whose most likely mass ranges between 0.15 and $0.9 M_{\odot}$ (cf. Fig. 4). Consistently, the EROS project (Lasserre et al.2000) found, based on observations of LMC and SMC, that MACHOs cannot dominate the Galactic halo if their masses are $\lesssim 1 M_{\odot}$. They find that the halo mass fraction in MACHOs is $<20 \%$ for MACHO masses between $10^{-7} M_{\odot}$ and $0.1 M_{\odot}$ (at $95 \%$ confidence).

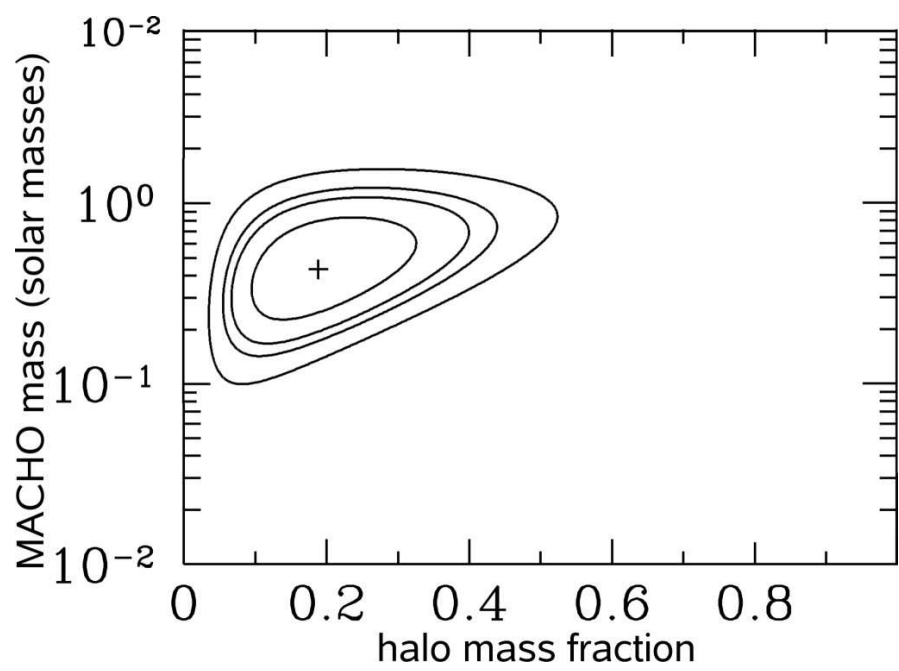

FIG. 4.-Example of likelihood contours obtained from the MACHO experiment for one specific model for the Milky Way halo. The abscissa is the fraction of the halo mass contained in MACHOs, the ordinate is the MACHO mass. The contours show the $60 \%, 90 \%, 95 \%$, and $99 \%$ confidence levels. (adapted from Alcock et al. 2000)

Thus, although MACHOs have been detected between us and the Magellanic Clouds, they are insufficient for explaining all of the Milky Way's dark mass. These MACHOs can in principle be anywhere between the source stars and the observer, i.e. in the dark halos of the Milky Way or of the Magellanic Clouds (Gould 1993, Sahu 1994; Wu 1994b; Gould 1995; Evans \& Kerins 2000). It had been speculated that self-lensing within the LMC might suffice for explaining the observed optical depth (Aubourg et al. 1999), but later studies showed that certainly not all of the LMC lensing events can be explained as being due to stars in the LMC. Rather, the LMC needs to be embedded into an extended halo (Gyuk et al. 2000; Jetzer et al. 2002; Mancini et al.2004). Microlensing experiments have thus confirmed that the Galaxy and the Magellanic Clouds must have extended dark halos, only a fraction of which can be composed of compact objects of stellar and sub-stellar mass.

Microlensing monitoring programs are now targeting the Andromeda galaxy M 31. Few early detections of microlensing candidates (Paulin-Henriksson et al. 2003; Riffeser et al. 2003) proved the feasibility of such surveys, and data are now accumulating beginning to allow constraining the halo fraction of MACHOs in M 31 (de Jong et al. 2004, Uglesich et al. 2004), supporting results consistent with those found in our Galaxy.

\subsection{Galaxies}

\subsubsection{Density profiles}

Well over 70 cases of strong lensing by galaxies are now known. Most of them have two or four images, but a few have higher image numbers. Image splittings, typically of order an arc second, allow the projected lens mass to be constrained which is enclosed by the images. However, it turns out to be surprisingly difficult to constrain the mass profile. Essentially, multiple images constrain the average surface-mass density in an annulus bounded by the images.

Using many lens systems, and assuming their mass profiles to be self-similar, it becomes possible to trace the average surface mass density at different radii, and thus to map out the density profile. Analysing 22 galaxy lenses, and adapting a mass model composed of a concentrated component representing the light and a power-law component representing the dark matter, Rusin et al. (2003) found that the slope of the density profile is very nearly isothermal, with a double-logarithmic slope of $n=2.07 \pm 0.13$ (isothermal has $n=2$; cf. Sect. 2.5; cf. Fig. 5 Models in which the mass traces the light and is therefore more centrally concentrated fail at the $99 \%$ confidence level. If the dark matter follows the NFW density profile, $(22 \pm 10) \%$ of the matter inside two effective radii has to be dark. They also find a weak trend of the mass-to-light ratio, $M / L \propto L^{0.14_{-0.12}^{+0.16}}$, consistent with the fundamental plane of elliptical galaxies. Treu \& Koopmans (2004) agree that the density profiles are nearly isothermal, but find a somewhat larger scatter. They confirm that lensing galaxies in which light traces mass are ruled out, and find a dark-matter fraction of between $15 \%$ and $65 \%$ within the effective radius.

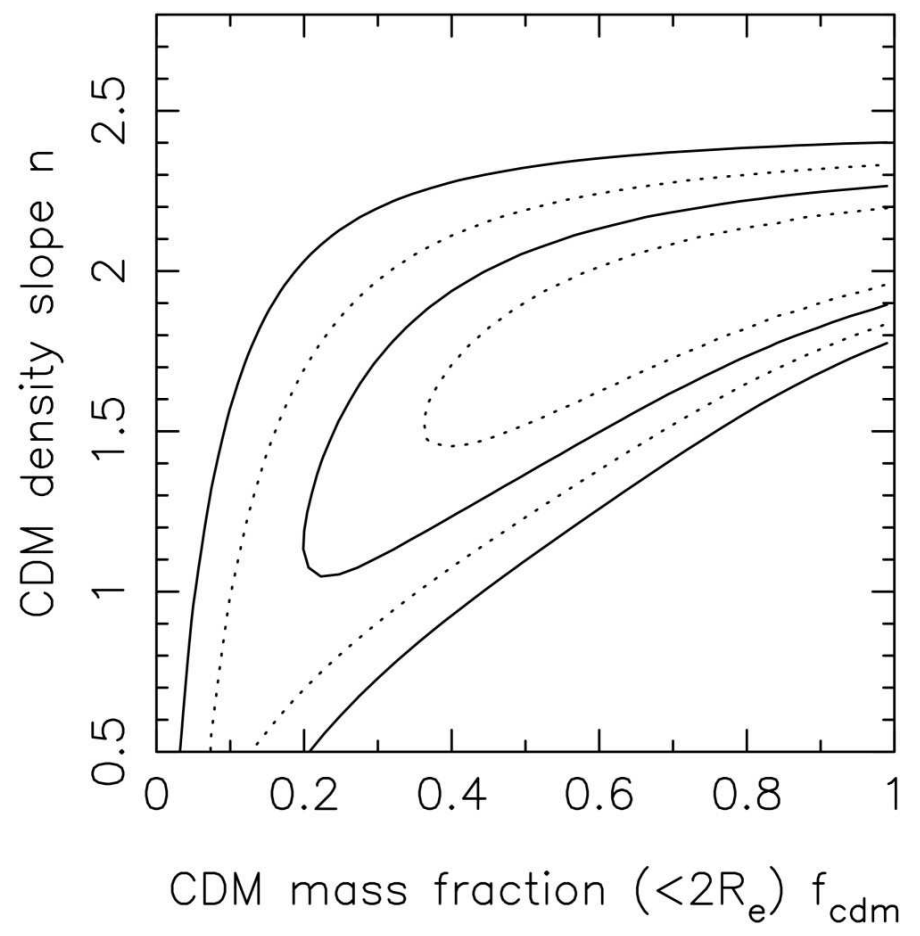

FIG. 5.-Likelihood contours in the plane spanned by the dark mass fraction in lensing galaxies (abscissa) and the slope of their density profile (ordinate). The solid and dotted contours show the $68 \%$ and $95 \%$ confidence levels for two and one free parameter, respectively. (adapted from Rusin et al. 2003)

We have seen in the introduction that gravitational lenses are expected to produce an odd number of images. In contrast, all 
but very few observed galaxy-lens systems have an even image number, most of them either two or four. The missing images are expected to be faint if the central density profile of the lensing galaxies is steep enough, thus their absence can be used for constraining the central concentration of the lensing mass distributions. Based on this argument, Rusin \& Ma (2001) find that inner mass distributions of lensing galaxies cannot be much shallower than isothermal. Conversely, Winn et al. (2004) use a lens system in which a faint, central image has been found to constrain the mass of the central black hole in the lensing galaxy to be $<2 \times 10^{8} h^{-1} M_{\odot}$. From the general absence of faint, central images, Keeton (2001) concludes that the central mass profiles of lensing galaxies must be more concentrated than CDM alone predicts. Central black holes may reconcile CDM density profiles with even image numbers only if they are about an order of magnitude more massive than expected from the relation between black-hole and bulge masses.

\subsubsection{Time delays and the Hubble constant}

Time-delay measurements in multiple-image systems promise constraints on the Hubble constant, provided a sufficiently accurate mass model for the lens is known. Conversely, considering the Hubble constant as known, time-delay measurements can be used as further constraints on the lensing density profile. Values for the Hubble constant derived this way tended to be lower than those, e.g. obtained from the HST Key Project (e.g. Fassnacht et al.2002), but lens models constructed upon a large number of constraints yield values which are very well in agreement with other determinations. For instance, Koopmans et al. (2003) find $H_{0}=75_{-6}^{+7} \mathrm{~km} \mathrm{~s}^{-1} \mathrm{Mpc}^{-1}$ from time delays measured in the quadruply lensed quasar B $1608+656$.

An interesting problem with interpreting time delays in galaxy-lens systems is pointed out by Kochanek (2003). It turns out that the time delays between multiple images is essentially determined by the mean surface-mass density in an annulus around the lens centre bounded by the images. An estimate for that mass density can also be obtained converting the visible light to mass, assuming typical values for the fraction $f_{\mathrm{b}}$ of matter that condenses into stars. Adopting $f_{\mathrm{b}} \simeq 0.02$ in accordance with local observations works well with near-isothermal mass models, but yields substantially too low values for the Hubble constant, $H_{0}=(48 \pm 5) \mathrm{km} \mathrm{s}^{-1} \mathrm{Mpc}^{-1}$. Conversely, values for the Hubble constant agreeing with the HST Key Project result, $H_{0}=(72 \pm 8) \mathrm{km} \mathrm{s}^{-1} \mathrm{Mpc}^{-1}$, are compatible with the measured time delays only if lens models with constant mass-to-light ratios are adopted, which are otherwise ruled out. There seems to be an as yet unexplained discrepancy between measurements of $H_{0}$ and the measured time delays within the CDM framework.

\subsubsection{Substructure in lensing galaxies}

Interestingly, the number of observed lenses with four images (quadruples) are about as abundant as such with two images (doubles), while they should only contribute $25 \%$ to $30 \%$ of the galaxy lenses. The fraction of quadruples can be enhanced by satellite galaxies orbiting the main lens galaxies (Cohn \& Kochanek 2004) or by matter in their larger-scale environments (Keeton \& Zabludoff 2004), although the latter explanation is potentially problematic because it also tends to lower inferred values of the Hubble constant.

Axially-symmetric lens models are insufficient for modelling observed multiple-image systems. At least elliptical lens models are necessary. Embedding the lenses into additional external shear fields helps fitting observed image configurations, but typically more shear is required $(10 \%-15 \%)$ than the average large- scale structure can provide (1\%-3\%, Keeton et al. 1997). This hints at the presence of angular structure in the lensing galaxies.

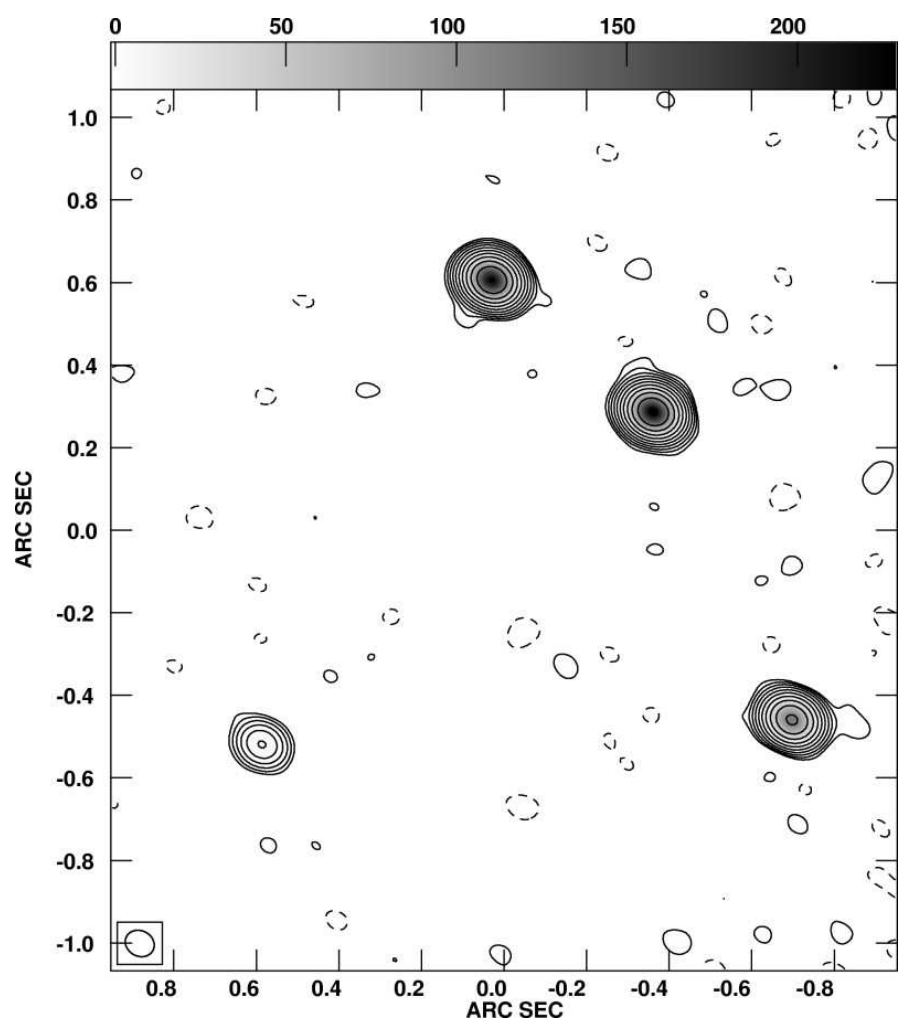

FIG. 6.-MERLIN radio image of the multiply-imaged quasar B $1422+231$ (courtesy of the JVAS/CLASS team). If image by a single, unperturbed lens, the two outer of the three bright images should together be as bright as the one between them.

It is an interesting problem which caused much recent discussion that lens models are typically very successful in reproducing image positions, but fail in a large fraction of lens systems to explain the flux ratios between different images. A particularly obstinate and well-known case is B 1422+231 (Kormann et al. 1994; Hogg \& Blandford 1994). This is most striking in situations where the source falls just inside a cusp, in which case the sum of the signed magnifications of the three related images should vanish exactly. This expectation is frequently violated in real lens systems.

Mao \& Schneider (1998) first suggested that substructure in the lensing galaxy could account for these anomalous flux ratios. While microlensing by the stars in the lens would less affect radio than optical fluxes because of their larger size, lensing by larger-scale substructures would equally change radio and optical flux ratios. Alternatively, CDM galaxy halos should contain sub-halos which may also account for anomalous flux ratios. Bradač et al. (2002) found similar modelling problems for images produced by a simulated lensing galaxy as for B $1422+231$. Chiba (2002) discussed that the sub-halo population of CDM halos produces perturbations of the magnitude required for explaining anomalous flux ratios. Metcalf \& Zhao (2002) estimated that $\gtrsim 5 \%$ of the lensing halo mass must be contained in substructures and argued that elliptically deformed power-law models embedded into external shear are insufficient for most lenses. Similarly, Dalal \& Kochanek (2002) concluded that substructure comprising $0.6 \%$ to $7 \%$ of the lens mass, with a median at $2 \%$, was necessary for reproducing the observed anomalous flux ratios, in excellent agreement with CDM halo simulations. They also estimated that the sub-halos should have masses in the 
range $\left(10^{6} \ldots 10^{9}\right) h^{-1} M_{\odot}$. Metcalf (2002) found that halo substructures with masses within $\left(10^{5} \ldots 10^{7}\right) h^{-1} M_{\odot}$ may explain the curved radio jet in B $1152+199$.

In contrast to these arguments, Evans \& Witt (2003) explicitly constructed smoothly deformed lens models which could well reproduce image configurations and flux ratios for most lens systems and argued that substructure in the lensing galaxies and smoothly deformed lenses are both viable explanations for the anomalous flux ratios. Along the same line, Möller et al. (2003) and Quadri et al. (2003) showed that disks in lens galaxies can alter image magnification ratios considerably, while Chen et al. (2003) pointed out that halos projected onto the main lens galaxy may also cause the observed magnification perturbations.

From a somewhat different perspective, Schechter \& Wambsganss (2002) discussed that decomposing lensing galaxies into microlenses has the most prominent effect when part of the lensing mass remains smooth. Specifically, they showed that socalled saddle-point images can be substantially demagnified in presence of microlenses. Schechter et al. (2004) added that the magnification distribution of the macro-images depends on the mass spectrum of the microlenses, in contrast to earlier expectations.

Kochanek \& Dalal (2004) investigated various alternative explanations for the anomalous flux ratios, such as absorption, scattering, scintillation, uncertainties in the macro-model, and stellar microlensing, and arrived again at the conclusion that halo substructures remain as the most likely reason. Bradač et al. (2004) verified that numerically simulated galaxies can produce anomalous flux ratios as observed and emphasised the importance of the demagnification of saddle-point images. However, the statistics remains unclear. Although it appears doubtless that CDM halos contain sufficient substructure for sufficiently perturbing image flux ratios, such sub-halos must also appear projected onto at least one of the images. The probability for that is low. Mao et al. (2004) find in numerical simulations that the probability of finding suitably massive sub-halos in front of macro-images is only $\lesssim 0.5 \%$, which is probably too low for explaining the anomalous flux ratios. The situation thus remains interestingly confused.

\subsubsection{Lens statistics}

The abundance of galaxy lenses has often been used for constraining the cosmological constant $\Lambda$. While early studies typically found upper limits of $\Lambda \lesssim 0.7$ (e.g. Kochanek 1996; Falco et al. 1998), more recent investigations find values which are better compatible with other determinations, (e.g. Chiba \& Yoshii 1999, Chae et al. 2002), finding spatially-flat model universes with low matter density $\left(\Omega_{0} \simeq 0.3\right.$ ) preferred. The reason for this change is that gradually more realistic galaxy luminosity functions were used for estimating the expected number of lenses, rather than error-prone extrapolations of local galaxy number densities towards high redshift (Keeton|2002).

Halos are expected to have a continuous mass spectrum in universes dominated by cold dark matter, which is described by mass functions such as those derived by Press \& Schechter (1974), Sheth \& Tormen (2002) and Jenkins et al. (2001). Thus, one would expect a continuous distribution of splitting angles between fractions of an arc second to several ten arc seconds. Narayan \& White (1988) investigated whether the observed image-splitting distribution was consistent with expectations from CDM. They found observation and theory by and large agreed if selection effects were taken into account. Kochanek (1995) found that the splitting-angle distribution in CDM grossly incompatible with microwave-background constraints in a model universe with high matter density and van- ishing cosmological constant, but that both could be comfortably reconciled in a spatially-flat, low-density CDM model.

Occasionally, therefore, lens systems should be detected with splitting angles of ten or more arc seconds. Phillips et al. (2001) interpreted the absence of wide-separation lenses in the CLASS survey as a being due to low central mass concentrations in group- and cluster-sized halos. It was perceived as a further confirmation of the CDM paradigm when a quadruply imaged quasar was detected in the Sloan Digital Sky Survey with a splitting angle of 14.62 arc seconds (Inada et al. 2003), for which Williams \& Saha (2004) derived a lens mass of $(5 \pm 1) \times 10^{13} h^{-1} M_{\odot}$ within a radius of $100 h^{-1} \mathrm{kpc}$ based on a non-parametric lens model. Oguri \& Keeton (2004) noted that the triaxiality of CDM halos must be taken into account in probability and mass estimates for the formation of wide-separation lens systems, a theme which is familiar from studies of strong lensing in galaxy clusters.

\subsubsection{Galaxy-galaxy lensing}

Less distant galaxies can act as weak gravitational lenses on more distant galaxies. Their shear imprints a feeble tangential distortion pattern on the images of background galaxies which appear projected close to them. This weak signal is superposed on the intrinsic ellipticities and irregularities of the background-galaxy images and thus requires statistical techniques for its extraction. Brainerd et al. (1996) first discussed the principal features of this effect and searched for it in a sample of galaxies, in which they separated background from foreground galaxies according to their apparent brightness. They could already infer that the shear profile of brighter galaxies was compatible with an isothermal mass profile with a circular velocity of $v_{\mathrm{c}}=(220 \pm 80) \mathrm{km} \mathrm{s}^{-1}$. They also placed a lower limit $r_{*} \gtrsim 100 h^{-1} \mathrm{kpc}$ on the halo size of the lensing galaxies. dell'Antonio \& Tyson (1996) searched for galaxy-galaxy lensing in the Hubble Deep Field (North) and found a mean velocity dispersion for the lensing halos of $\sigma_{\mathrm{v}}=185_{-35}^{+30} \mathrm{~km} \mathrm{~s}^{-1}$, and a weak lower limit on the halo radius.

Schneider \& Rix (1997) devised a maximum-likelihood technique for efficient analysis of galaxy-galaxy lensing data which specifically took the redshift distributions of foreground and background galaxies into account. They applied this technique to numerically simulated data and calibrated its performance. Natarajan \& Kneib (1997) and Geiger \& Schneider (1998) developed methods for detecting the weak-lensing signal of galaxies embedded in galaxy clusters. Applying their technique to the cluster $\mathrm{Cl}$ 0939+4713, Geiger \& Schneider (1999) detected the shear signal of individual massive cluster galaxies. More recently, Natarajan et al. (2002a) compared the weak-lensing signal of early-type, $L_{*}$ galaxies in clusters and in the field and found evidence for the cluster galaxies to be truncated, with a truncation radius shrinking with the density of the environment. Hoekstra et al. (2004) combined weak-lensing data on galaxy halos to show that they are flattened.

Recent wide-field surveys also triggered an exciting development of galaxy-galaxy lensing. Fischer et al. (2000) used the Commissioning Data of the SDSS to infer that the tangential shear profile is compatible with a power law with exponent between 0.7 and 1.1 , i.e. close to isothermal. They found a best-fitting circular velocity of $v_{\mathrm{c}}=(150 \ldots 190) \mathrm{km} \mathrm{s}^{-1}$ and a lower limit to the physical halo radius of $260 h^{-1} \mathrm{kpc}$. From the Las Campanas Redshift Survey, Smith et al. (2001) deduced an isothermal tangential shear profile within $200 h^{-1} \mathrm{kpc}$ and a circular velocity of $v_{\mathrm{c}}=(164 \pm 20) \mathrm{km} \mathrm{s}^{-1}$ for $L_{*}$ field galaxies. They found a virial mass for the dark halo of a typical $L_{*}$ galaxy 
of $(2.7 \pm 0.6) \times 10^{11} h^{-1} M_{\odot}$. Wilson et al. (2001) used data taken with the UH8K camera at the Canada-France-Hawaii telescope to measure galaxy-galaxy lensing. They also found tangential shear profiles compatible with an isothermal slope and a rotation velocity of $v_{\mathrm{c}}=238_{-30}^{+27} \mathrm{~km} \mathrm{~s}^{-1}$ for $L_{*}$ galaxies. They concluded that the mass-to-light ratio of $L_{*}$ galaxies in the $B$ band is $M / L \simeq(121 \pm 28) h L_{\odot} / M_{\odot}$.

Combining the galaxy-galaxy weak-lensing signal obtained from SDSS data with the Tully-Fisher and fundamental-plane relations for late- and early-type galaxies, respectively, Seljak (2002) found that the galaxy velocity profile must drop substantially towards the virial radius, which indicates a steep darkmatter profile. Guzik \& Seljak (2002) compared theoretically motivated CDM halo models with SDSS data and constrained the halo properties of galaxies with luminosities $\gtrsim L_{*}$. They constrained the virial mass of an early-type $L_{*}$ halo to $M_{200}=$ $(5 \ldots 10) \times 10^{11} h^{-1} M_{\odot}$, and somewhat less for late-type galaxies, depending on the colour. They found a gentle increase of the mass-to-light ratio with luminosity, with $M / L \simeq 17 h M_{\odot} / L_{\odot}$ for late-type and $M / L \simeq 45 h M_{\odot} / L_{\odot}$ for early-type $L_{*}$ galaxies.

Sheldon et al. (2004) studied the cross-correlation between galaxies and mass from the galaxy-galaxy lensing signal detected in SDSS data. The wide area covered by the survey allowed constraining the correlation function out $10 \mathrm{~h}^{-1} \mathrm{Mpc}$. They find a power law with a correlation length of $r_{0} \simeq(5.4 \pm$ 0.7) $h^{-1} \mathrm{Mpc}$ and an exponent of $1.79 \pm 0.05$. The bias parameter turns out to be approximately scale-independent (see also Hoekstra et al. 2001), while Hoekstra et al. (2002b) find the bias parameters to be gently increasing from Mpc to larger scales. Comparisons with theoretical expectations for the galaxy distribution relative to the dark matter find overall good agreement (Weinberg et al. 2004), except that the simulated mass-to-light ratio is somewhat too high (Yang et al. 2003). Satellite galaxies orbiting the lensing galaxies could be physically aligned with their hosts and thus mimic a weak galaxy-galaxy lensing signal. Hirata et al. (2004) estimated this possible contamination and constrained it to less than $15 \%$ at the relevant scales.

\section{Galaxy Clusters}

\subsection{Strong lensing}

\subsubsection{Qualitative conclusions}

Strong lensing in galaxy clusters was first detected by Soucail et al. (1987) and Lynds \& Petrosian (1989). They found extended, arc-like images in the galaxy clusters A 370 and Cl 2244. Several explanations were proposed for these objects, among them gravitational lensing of background galaxies (Paczyński 1987), which was confirmed when the redshift of the arc in A 370 was measured and found to be substantially higher than the cluster's (Soucail et al. 1988).

It was quickly recognised that gravitational arcs provided important information on the structure of galaxy clusters. It was unclear at the time how the dark matter was distributed and whether the X-ray surface-brightness profiles, which typically show a flat core of $\simeq 200 h^{-1} \mathrm{kpc}$ radius, were representative for the dark-matter profiles. Arcs were soon found to reveal the following about clusters: (1) Cluster mass distributions cannot typically be axially symmetric, because large counter-arcs would otherwise be expected (Grossman \& Narayan 1988, Kovner 1989). (2) The substantial amounts of dark matter in galaxy clusters cannot be attached to the galaxies because arcs would then have much smaller curvature radii (Hammer et al. 1989 , Bergmann et al. 1990). Particularly striking were the detections of "straight arcs" in two clusters (Pelló et al.|1991, Mathez et al.

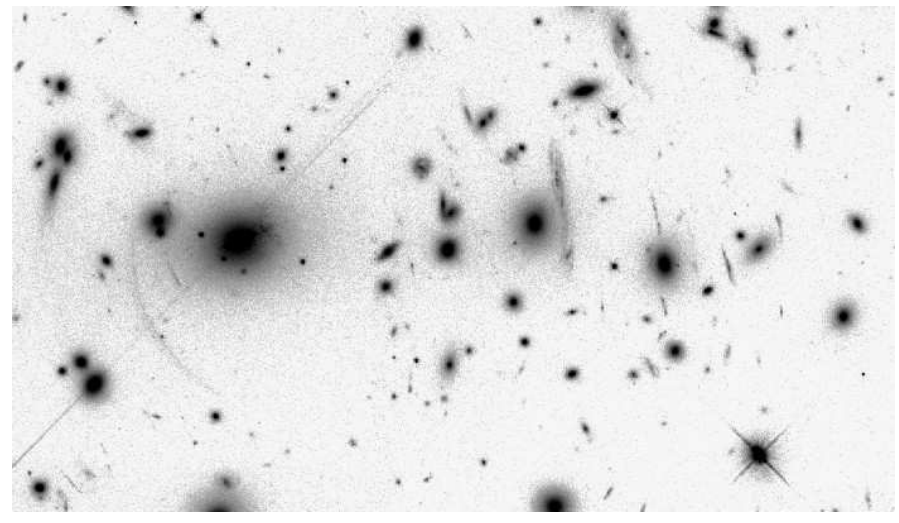

FIG. 7.-HST image of the cluster Abell 2390 (courtesy Jean-Paul Kneib). The "straight arc" near the centre directly demonstrates the presence of substantial amounts of dark matter in this cluster.

1992, Pierre et al. 1996) because they visually demonstrated the need for substantial concentrations of dark matter with very high mass-to-light ratio (Kassiola et al.1992). (3) Clusters need to have steep density profiles, because arcs would be substantially thicker otherwise (Hammer \& Rigaut 1989). For clusters to be strong lenses, their central convergence $\kappa$ has to be close to unity, but for arcs to be thin, the convergence at their locations has to be around 0.5 . From cluster centres to the arc radii of typically $10^{\prime \prime} \ldots 30^{\prime \prime}$, the $\kappa$ profile must thus fall by approximately a factor of two. Cluster core radii, if they exist, must thus be substantially smaller than the X-ray core radii, which was also confirmed by the detection of "radial arcs" (Fort et al. 1992, Miralda-Escudé 1993, Mellier et al. 1993).

\subsubsection{Cluster masses}

Arcs allow cluster masses to be easily estimated. It was soon discovered that the masses obtained this way are very close to mass estimates derived from the X-ray temperature and surfacebrightness profile. This is not obvious because gravitational lensing is sensitive to the mass in whatever form and physical state it may be, while the interpretation of $\mathrm{X}$-ray data requires assumptions on symmetry and hydrostatic equilibrium of the gas with the gravitational potential well, if not on isothermality of the intracluster gas. This being reassuring, a systematic discrepancy was soon revealed in the sense that masses derived from strong lensing were typically higher by factors of $\simeq 2 \ldots 3$ than X-ray masses ( $\mathrm{Wu} 1994 \mathrm{a}$; Miralda-Escudé \& Babul 1995; Wu \& Fang 1996); more recent examples are, e.g. Chen et al. (2003) and Ota et al. (2004).

Bartelmann \& Steinmetz (1996) used numerical simulations to show that X-ray mass estimates can be systematically lower in merging clusters because their X-ray gas is still cooler than expected from their total mass, which is already seen by the lensing effect. This seems to explain the mass discrepancy at least in some clusters (e.g. Smail et al. 1995; Ota et al.1998). Asymmetries and cluster substructures also play an important role. Due to their relatively larger shear, asymmetric and substructured clusters are more efficient lenses at a given mass. Mass estimates based on axially symmetric models are thus systematically too high (Bartelmann 1995a; Hattori et al. 1998).

Allen (1998) distinguished clusters with and without cooling flows and found an appreciable mass discrepancy in clusters without, but good agreement of X-ray and lensing mass estimates in clusters with cooling-flow. This supports the concept that well-relaxed clusters which had sufficient unperturbed time to develop a cooling flow are well-described by simple, axially- 
symmetric models for lensing and the X-ray emission, while dynamically more active clusters tend to give discrepant mass estimates; this was confirmed by $\mathrm{Wu}(2000)$. Makino \& Asano (1999) noted that the mass discrepancy is reduced if cluster density profiles are steeper than inferred from the X-ray emission.

\subsubsection{Cluster mass profiles}

Assuming mass profiles with cores, tangential arcs require small core radii as described above, but radial arcs require the cores to be finite (Le Fevre et al. 1994; Luppino et al. 1999). Numerical simulations of CDM halos, however, show that density profiles flatten towards the core, but do not develop flat cores (Navarro et al. 1996, 1997). Bartelmann (1996) showed that radial arcs can also be formed by halos with such "cuspy" density profiles, provided the central cusp is not too steep.

In principle, the relative abundances and positions of radial compared to tangential arcs in clusters provide important constraints on the central density profile in clusters (Miralda-Escudé 1995: Molikawa \& Hattori 2001; Oguri et al. 2001). Radial arcs are still too rare for successfully exploiting this method. Being much closer to the cluster cores than tangential arcs, they are also more likely to be confused with or hidden by the light of the cluster galaxies. Following Miralda-Escudé (1995), Sand et al. (2004) compiled a sample of clusters containing radial and tangential arcs and added constraints on the central mass profile from velocity-dispersion measurements in the central cluster galaxies. They demonstrated that, assuming axially-symmetric mass distributions, central density profiles have to be substantially flatter than those found in CDM simulations. However, Bartelmann \& Meneghetti (2004) showed that even small deviations from axial symmetry can invalidate this conclusion and establish agreement between these observations and CDM density profiles.

Attempts at modelling arcs with isothermal mass distributions are typically remarkably successful (see Kneib et al. (1996) for an impressive example). This is all the more surprising as numerical simulations consistently find density profiles which are flatter than isothermal within the scale radius and steeper outside. In a very detailed analysis, Gavazzi et al. (2003) find that an isothermal core profile for the cluster MS 2137 is preferred compared to the flatter NFW profile. Smith et al. (2001) constrain the core density profile in A 383 using X-ray, weak-, and strong-lensing data and find it more peaked than the NFW profile, but argue that this may be due to the density profile of the cD galaxy. Conversely, Kneib et al. (2003) find in a combined weak- and strong-lensing analysis of $\mathrm{Cl} 0024+1654$ that an isothermal mass profile can be rejected, while the NFW profile fits the data well. The most likely explanation is that the innermost cluster density profiles can be significantly influenced and steepened by baryonic physics.

\subsubsection{Arc abundance and statistics}

The mean density profile of galaxy clusters can also be constrained statistically because the probability for a cluster to become a strong lens depends sensitively on the mass concentration in its core (Wu \& Hammer 1993). Miralda-Escudé (1993) suggested that the core densities of strong-lensing clusters could be enhanced by projection of elongated clusters along the lineof-sight. Bartelmann \& Weiss (1994) used a numerically simulated galaxy cluster to show that asymmetric, substructured cluster models are significantly more efficient strong lenses than axially-symmetric mass distributions because of their substantially larger shear field. Averaging over a sample of simulated clusters, Bartelmann et al. (1995) quantified that the cross sections for arc formation could be up to two orders higher for asymmetric than for axially symmetric cluster models of the same mass.

Hattori et al. (1997) confirmed that structured lenses help understanding the observational results of Le Fevre et al. (1994), who detected six arcs in a sample of 16 clusters selected for their high X-ray luminosity as measured by the EMSS satellite, but argued that even more concentrated mass profiles than those used by Bartelmann et al. (1995) are necessary for explaining them quantitatively. Bartelmann et al. (1998) used samples of numerically simulated clusters to estimate the total arcformation probability in different cosmological models. Comparing their results with the data from Le Fevre et al. (1994), they concluded that only their cluster sample taken from a simulation with low matter density $\left(\Omega_{0}=0.3\right)$ and no cosmological constant could well reproduce the measured high arc abundance, but the other three models failed badly. In particular, a flat cosmological model with $\Omega_{0}=0.3$ and $\Omega_{\Lambda}=0.7$ produced an order of magnitude less arcs than observed.

This so-called arc-statistics problem was disputed based on calculations using analytic models for cluster lenses (Cooray 1999, Kaufmann \& Straumann 2000), which failed to reproduce the strong dependence on the cosmological constant claimed by Bartelmann et al. (1998). The possible influence of cluster galaxies on the arc-formation efficiency of cluster lenses was investigated by Flores et al. (2000) and Meneghetti et al. (2000), but found to be negligible. Molikawa et al. (1999) confirmed that axially-symmetric mass models adapted to the X-ray emission do not produce a sufficient number of arcs. They found that using NFW profiles for the dark-matter profile helped, but the profiles required too high masses, and proposed that substructured mass distributions could be the solution. Meneghetti et al. (2003b) adapted elliptically distorted lenses with NFW mass profile (see also Golse \& Kneib 2002) to numerically simulated clusters and found the analytic models inadequate for quantitative arc statistics despite the asymmetry, demonstrating the importance of substructures.

Oguri et al. (2003) studied the strong-lensing properties of triaxial (rather than ellipsoidal) halos and found that they may well explain the high arc abundance (Le Fevre et al. 1994; Luppino et al. 1999), provided their central density slopes are steep enough, with a double-logarithmic slope near -1.5 . Wambsganss et al. (2004) simulated the magnification probability for light rays propagating through a section of the Universe and found that the abundance of high-magnification events depends strongly on the source redshift. They attributed this to the exponential mass function of massive halos, which leads to a steep increase with source redshift in the number of halos suitable for strong lensing. Identifying the probability for highly magnified light bundles on random patches of the sky with the probability for finding arcs in massive galaxy clusters, they suggested this result as the resolution for the arc statistics problem. Dalal et al. (2004) used numerical cluster simulations to estimate arc cross sections and found reasonable agreement with the earlier results of Bartelmann et al. (1998), but arrived at a higher expected arc abundance because they inserted a higher normalisation for the number density of both X-ray clusters and background sources.

Williams et al. (1999) noted that the arc radii in clusters depend only weakly on clusters mass and suggested that massive $\mathrm{cD}$ galaxies may be the reason. However, Meneghetti et al. (2003a) studied the effect of cD galaxies on the overall arc abundance and found it insufficient to remove the arc statistics problem. If the cosmological constant is replaced by some form of dynamical dark energy, structures tend to form earlier during cosmic history. Since cluster core densities reflect the mean cosmic density at their formation time, clusters thus tend to be more concentrated in dark-energy compared to cosmological-constant 
models. Bartelmann et al. (2003) estimated the effect of higher cluster concentrations on arc statistics by analytic means. They found that dark energy may in fact increase arc abundances noticeably, but again not sufficiently for solving the arc statistics problem.

The problem has experienced an interesting recent twist. Galaxy clusters at high redshifts are found to be remarkably efficient lenses (Gladders et al. 2003, Zaritsky \& Gonzalez 2003) even though they should be by far not massive enough for producing large arcs. A particularly impressive example is the cluster RX J105343+5735 at $z=1.263$ which contains a large arc from a source at $z=2.577$ (Thompson et al. 2001). In this respect, it is interesting that the strong-lensing efficiency of clusters can be increased substantially and on a short timescale during a major merger (Torri et al. 2004). As a subcluster approaches a cluster, the tidal field is increased, leading to a first maximum of the cross section approximately when the two virial regions touch. The cross section then slightly decreases and approaches a second maximum when the separation of cluster and subcluster is minimal. A third peak corresponding to the first is formed when the subcluster leaves the virial region again after the merger. During that process, the arc cross section can change by an order of magnitude or more on a time scale of $\simeq 0.1 \mathrm{Gyr}$. It thus appears that strong lensing can be a transient phenomenon at least in some clusters which would otherwise be not massive or concentrated enough. The dependence of the main merger epoch on cosmic history would then establish an interesting link between high-redshift, strong cluster lenses and the cosmological framework model.

\subsection{Other applications of strong cluster lensing}

If a cluster produces arcs from multiple sources at different redshifts, the lensing mass distribution remains the same, but the geometrical lensing efficiency is different for the arcs. Since this depends on cosmological parameters, these can thus be purely geometrically constrained from multiple-arc systems (Link \& Pierce 1998, Gautret et al. 2000). Soucail et al. (2004) applied this technique to multiple arcs in the cluster A 2218 and found that a universe with critical matter density and no cosmological constant is excluded at $>4 \sigma$ confidence from this single cluster.

For alleviating potential problems e.g. with the abundance of satellite galaxies, it was proposed that the dark-matter particles might interact with each other in another way than through gravity. Such a self-interaction would act as a source of isotropic pressure and thus symmetrise and smooth mass distributions (Miralda-Escudé 2002). Strong gravitational lensing, being very sensitive to cluster asymmetries, places a tight limit on the interaction cross section. Using numerical simulations, Meneghetti et al. (2001) showed that the strong-lensing efficiency of galaxy clusters would abruptly disappear if the specific self-interaction cross section was $\gtrsim 0.1 \mathrm{~cm}^{2} \mathrm{~g}^{-1}$.

Finally, lensing clusters are frequently being used as cosmic telescopes, magnifying distant sources above the limits for photometry or spectroscopy. To give a few examples, Casoli et al. (1996) used the magnification by A 370 to detect CO lines in arc sources; Frye \& Broadhurst (1998) and Pelló et al. (1999) identified sources at $z=4.04$ lensed by A 2390, Kneib et al. (2004) found an object with $z \simeq 7$ lensed by A 2218, and Pelló et al. (2004) claim to have detected an object with $z=10.0$ magnified by A 1835 .

\subsection{Weak cluster lensing}

\subsubsection{Cluster inversion}

Apart from the occasional spectacular strong-lensing effects, clusters imprint a coherent weak distortion pattern onto the many faint and distant galaxies in their background. Those galaxies reach number densities of $\simeq 40$ per square arc minute in typical images taken with large ground-based telescopes. The virial region of a typical galaxy cluster thus covers of order $10^{3}$ galaxies. Due to their intrinsically irregular shapes, lensinginduced distortions cannot be inferred from individual galaxies. Averaging over a few galaxies, however, the intrinsic ellipticities should average to zero, leaving the ellipticity caused by the gravitational shear as the average signal.

As shown in the introduction, shear and convergence are both related through the scalar lensing potential. Knowing the shear thus allows the scaled surface-mass density to be reconstructed. Kaiser \& Squires (1993) were the first to show that cluster convergence maps could be obtained by convolving the measured shear signal with a simple kernel, opening the way to systematic, parameter-free, two-dimensional cluster studies. Their technique was immediately applied to the cluster MS 1224, for which Fahlman et al. (1994) found a surprisingly high mass-tolight ratio of $\simeq 800 h M_{\odot} / L_{\odot}$ in solar units, about a factor of four times the typical cluster value.

Weaknesses in the convolution algorithm by Kaiser \& Squires (1993), such as its limitation to formally infinite data fields and weak shear, were discussed and removed by, e.g. Seitz \& Schneider (1995, 1996). Another technique for recovering cluster mass maps based on a maximum-likelihood approach was proposed by Bartelmann et al. (1996), and later augmented with maximum-entropy regularisation (Seitz et al. 1998) and further developed by Marshall et al. (2002). An algorithm for measuring the weak shear signal from data fields was described and implemented by Kaiser et al. (1995).

\subsubsection{Distributions of mass and light}

These inversion techniques for cluster lenses have by now been applied to numerous clusters. For most of them, the mass-tolight ratios turned out to be quite normal, i.e. $M / L \simeq 250 \ldots 300$ in blue and $M / L \simeq 150 \ldots 200$ in red colour bands, respectively. Some examples are Clowe et al. (1998); Hoekstra et al. (2002a) and Gavazzi et al. (2004). The high mass-to-light ratio found by Fahlman et al. (1994), however, was qualitatively confirmed by Fischer (1999). Thus, mass and light generally appear well correlated in weak-lensing clusters, although some interesting deviations have been observed. For instance, while mass follows light well in two of the three clusters in the A 901/902 supercluster field, the third cluster shows a significant offset between the mass and the light (Gray et al. 2002; see Fig. 8).

Similar phenomena appear in comparisons between the X-ray surface-brightness and the weak-lensing mass contours. While the X-ray emission follows the mass in many clusters (see Gioia et al. (1999); Clowe et al. (2000); Clowe \& Schneider (2002); Hoekstra et al. (2000) for examples), interesting deviations have recently been discovered. Machacek et al. (2002) find good agreement between surface-density and X-ray contours in the outer parts of A 2218, but deviations near the cluster centre, which they interpret as a sign of dynamical activity in the cluster. Several recent studies find the X-ray gas lagging behind the dark matter in merging clusters (Clowe et al. 2004; Markevitch et al. 2004, Jee et al. 2005), as expected for hot gas embedded into collision-less dark-matter halos.

If the dark-matter particles interacted with each other, such a separation between gas and dark matter would be suppressed. 


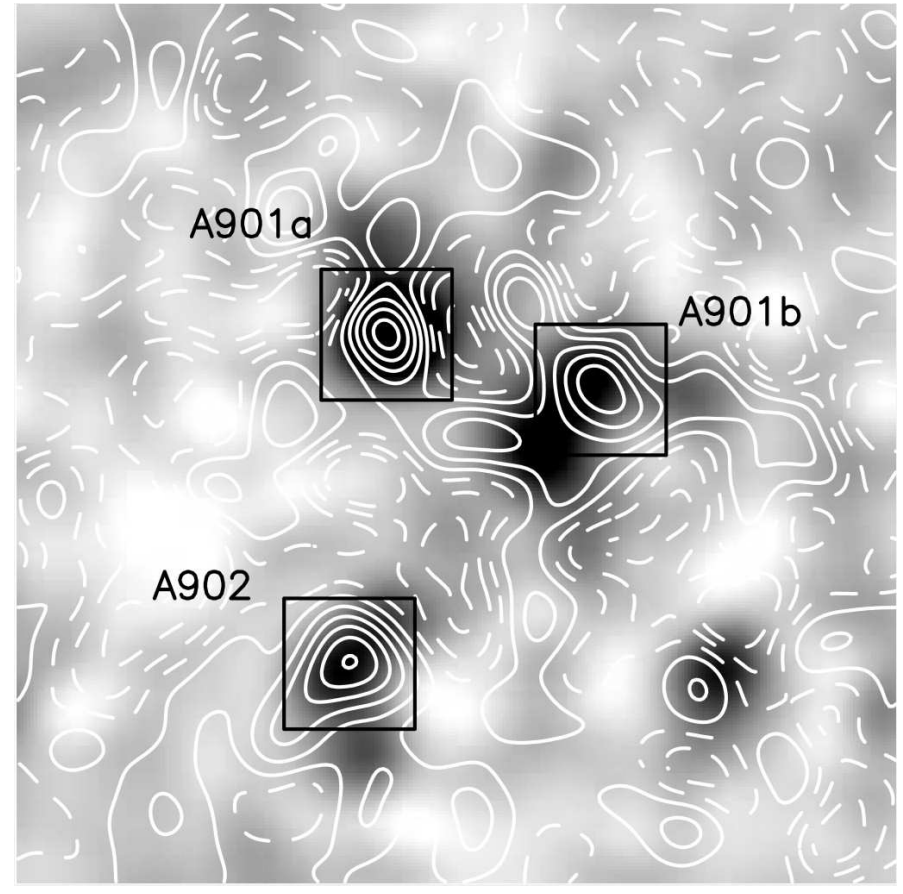

FIG. 8.- The supercluster field around A 901/902. The contours indicate the reconstructed convergence $\kappa$, the grey scale shows the smoothed distribution of the luminosity of early-type galaxies. (adapted from Gray et al. 2002)

Thus, from gas lagging behind the dark matter in merging clusters, and from small dark-matter core radii, limits could be obtained for the self-interaction cross section of the dark-matter particles, typically finding values $\lesssim(0.1 \ldots 1) \mathrm{cm}^{2} \mathrm{~g}^{-1}$ (Arabadjis et al. 2002, Natarajan et al. 2002b, Markevitch et al. 2004), comparable to what Meneghetti et al. (2001) concluded from strong cluster lensing. Although different mass estimates agree well in some clusters (e.g. Clowe \& Schneider 2002; Irgens et al. 2002; Jia et al. 2004, Jee et al. 2005, Margoniner et al. 2005), significant discrepancies between cluster masses derived from weak lensing and X-ray observations are frequently found (Lewis et al. 1999; Athreya et al. 2002, Holden et al. 2002) and interpreted as signalling dynamical processes in unrelaxed cluster cores. Of the 38 clusters in the X-ray selected sample studied by Dahle et al. (2002), $\simeq 30 \%$ show signs of dynamical activity, and more than $50 \%$ are strong lenses. Based on a sample of 24 clusters between redshifts 0.05 and 0.31 , Cypriano et al. (2004) claim that clusters with temperatures $\lesssim 8 \mathrm{keV}$ show good agreement between different mass estimates, while hotter clusters do not.

\subsubsection{Cluster mass profiles}

Projected cluster mass profiles obtained from weak lensing are often well fit by the isothermal profile (Sheldon et al. 2001) or by both the isothermal and the NFW mass profiles (Clowe et al. 2000, Clowe \& Schneider 2001; Athreya et al. 2002), while some clusters prefer NFW fits (Clowe \& Schneider 2001). NFW concentration parameters tend to be somewhat lower than theoretically expected (Clowe \& Schneider 2002; Hoekstra et al. 2002a; Jee et al. 2005), which may be due to intrinsically triaxial cluster halos (Clowe \& Schneider 2002). However, there is an increasing number of clusters for which NFW profiles with reasonably high concentration parameters are deduced (e.g. Clowe \& Schneider 2001; Arabadjis et al. 2002). Clowe et al. (2000) find the more massive of six high-redshift clusters less concen- trated than the less massive ones, which is also expected from theory. Dahle et al. (2003) fit the generalised NFW profile to six massive clusters at $z \simeq 0.3$, finding a central double-logarithmic slope $\alpha=-0.9 \ldots-1.6$ at $68 \%$ confidence. Assuming $\alpha=-1$, the concentration parameters are well in the expected range, i.e. 5 .. 10 depending on cluster mass.

Large-scale structure in front of and behind galaxy clusters is projected onto them and can affect weak-lensing mass determinations. Using large-scale structure simulations, Metzler et al. (1999) estimate that weak-lensing mass estimates exceed real cluster masses by several tens of per-cents due to the added large-scale structure. Hoekstra (2003) estimated that projected large-scale structure approximately doubles the error budget for weak-lensing cluster mass estimates. However, cluster mass profiles are affected by cluster substructures and asymmetries only at the per-cent level (King et al. 2001, Clowe et al. 2004).

We have seen in the discussion of strong cluster lensing that clusters at moderate and high redshifts, $z \gtrsim 0.8$, are already remarkably efficient strong lenses. The first weak-lensing mass map of a cluster at such high redshift (MS 1054-03 at $z=0.83$ ) was produced by Luppino \& Kaiser (1997). The weak-lensing signal of many similarly distant clusters was measured since, typically confirming the presence of well-developed, massive and compact clusters at that epoch (Clowe et al. 1998, Gioia et al. 1999: Margoniner et al. 2005), but also frequently indicating violent dynamical activity in cluster cores (Hoekstra et al. 2000, Holden et al. 2002, Jee et al. 2005).

\subsubsection{Dark clusters?}

Occasional detections of clusters with very high mass-to-light ratios (e.g. Fahlman et al. (1994); Fischer (1999); Gray et al. (2002) as discussed above) raise the question whether clustersized dark-matter halos may exist which are so inefficient in producing stellar or X-ray emission that they are invisible for anything but gravitational lensing. Erben et al. (2000) detected a peak in the weak-lensing signal 7 arc minutes south of the cluster A 1942 where no optical or infrared emission could be found (Gray et al. 2001). A similarly dark weak-lensing signal peak was discovered next to the high-redshift cluster $\mathrm{Cl} 1604+4304$ by Umetsu \& Futamase (2000). Another tangential shear alignment potentially revealing a dark halo (Miralles et al.2002) was meanwhile found to be spurious (Erben et al. 2003).

Schneider (1996) introduced the aperture-mass statistic specifically for detecting dark-matter halos through their weaklensing signal. The aperture mass is a weighted integral within a circular aperture over the shear component tangentially oriented with respect to the aperture centre. When applied to numerical simulations, the aperture-mass statistic turned out to be highly efficient in finding group- and cluster-sized halos, although the completeness of the resulting halo catalogues has to be balanced against the frequency of spurious detections by carefully choosing the signal-to-noise threshold (Reblinsky \& Bartelmann 1999, White et al. 2002). Wittman et al. (2001) report the first detection of a galaxy cluster through weak lensing, which was confirmed later through its optical signal. Conversely, Schirmer et al. (2004) use the aperture-mass technique for confirming the weak-lensing signal of clusters found optically in the ESO Imaging Survey.

Bartelmann et al. (2001) showed that the detection efficiency of the aperture-mass technique varies strongly with the density profile of the dark-matter halos, allowing a statistical discrimination between isothermal and NFW profiles. Miyazaki et al. (2002) found $4.9 \pm 2.3$ dark-matter halos in a field of 2.1 square degrees taken with the Subaru telescope, which is consistent with expectations based on CDM models and NFW density pro- 
files (Kruse \& Schneider 1999).

As mentioned before, cosmological models with dynamical dark energy cause dark-matter halos to be more concentrated compared to models with cosmological constant. While this should in principle lead to a higher number of weak-lensing halo detections in dark-energy cosmologies and thereby provide a way for discriminating cosmological-constant from dark-energy models, the expected sensitivity is very weak due to competing effects (Bartelmann et al. 2002; Weinberg \& Kamionkowski 2003). Weinberg \& Kamionkowski (2002) argue that clusters in formation, which are not virialised yet and thus under-luminous, may be detected through weak lensing. They suggest this as an explanation for the potential dark clusters found by Erben et al. (2000) and Umetsu \& Futamase (2000) and argue that cosmological constraints could be placed by comparing the numbers of visible and dark clusters.

\section{LARGE-SCALE STRUCTURES}

Weak gravitational lensing by large-scale structures is covered by several dedicated reviews, highlighting different aspects of this rich and quickly developing subject (Mellier 1999; Bartelmann \& Schneider 2001, Hoekstra et al. 2002c; Mellier \& van Waerbeke 2002; Refregier 2003). We can only summarise the most important aspects here and refer the interested reader to those reviews for further detail.

\subsection{Expectations and measurements}

Being inhomogeneously distributed in the Universe, matter on scales even larger than galaxy clusters must also gravitationally lens background sources. Early studies (Blandford et al. 1991; Miralda-Escudé 1991; Kaiser 1992) calculated the ellipticities and ellipticity correlations expected to be imprinted on the images of background galaxies, and found them to be of order a few per cent on arc-minute angular scales. In a first attempt at measuring this weak cosmological lensing signal, Mould et al. (1994) could place an upper limit in agreement with theoretical expectations.

Since weak cosmological lensing is highly sensitive to the non-linear evolution of the large-scale structures (Jain \& Seljak 1997), numerical simulations had to be carried out for precisely estimating the amplitude of the signal and the shape of the ellipticity correlation function (e.g. Bartelmann \& Schneider 1992 Jain et al. 2000, Hamana \& Mellier 2001; Vale \& White 2003). The cosmological potential of large weak-lensing surveys was quickly pointed out (Bernardeau et al. 1997; Kaiser 1998, van Waerbeke et al. 1999), emphasising the possibility of measuring in particular the matter density parameter $\Omega_{0}$ and the amplitude $\sigma_{8}$ of the dark-matter power spectrum.

Schneider et al. (1998) announced the detection of a coherent shear signal in the field of the radio galaxy PKS 1508-05 which they interpreted as being caused by large-scale structure lensing. The breakthrough came soon thereafter, when several different groups almost simultaneously reported the measurement of the cosmic-shear correlation function (Bacon et al. 2000; Van Waerbeke et al. 2000, Wittman et al. 2000, Maoli et al. 2001). Given the difficulty of the measurement and the different telescopes, cameras, and analysis techniques used, the agreement between these results and their compatibility with theoretical expectations was exciting and encouraging.

Cosmological parameters were soon derived from these first cosmic-shear measurements (Van Waerbeke et al. 2001b), finding $\sigma_{8} \gtrsim 0.7$ and $\Omega_{0} \lesssim 0.4$ for spatially-flat cosmological models. Two-point statistics of the cosmic shear are approximately proportional to the product $\sigma_{8} \Omega_{0}^{2}$, i.e. they are degenerate in these two parameters. This degeneracy can be lifted using third-order statistics such as the skewness (Van Waerbeke et al. 2001a), which arises because the non-linear evolution of cosmic structures leads to non-Gaussianity in the weak-lensing signal. Non-Gaussianities were first detected by Bernardeau et al. (2002) in the Virmos-Descart weak-lensing survey.

Much effort was devoted to calibrating weak-lensing measurements, to designing optimal cosmic-shear estimators and studying their noise properties. Erben et al. (2001) used numerical simulations to show that relative accuracies of $10 \% \ldots 15 \%$ can be reached by cosmic-shear measurements. A method for estimating the weak-lensing power spectrum inspired by the CMB data analysis was proposed by $\mathrm{Hu} \&$ White (2001). Cooray \& Hu (2001) investigated how non-Gaussianity can affect parameter estimates from the cosmic-shear power spectrum. Different estimators for the two-point statistics of cosmic shear and their correlation matrices were dicussed by Schneider et al. (2002a).

Numerous weak-lensing surveys have meanwhile been conducted. A non-exhaustive selection of the results obtained on $\sigma_{8}$ for fixed $\Omega_{0}=0.3$ is given in Tab. 1 . Although most values of $\sigma_{8}$ agree within the error bars, the scatter is still substantial. This is at least partially due to remaining systematics in the data analysis, as will now be discussed.

TABLE 1.-Non-exhaustive selection of results for $\sigma_{8}$ extracted from weak-lensing surveys. $\Omega_{0}=0.3$ is adopted throughout.

\begin{tabular}{|ll|l|}
\hline$\sigma_{8}$ & & reference \\
\hline 1.04 & \pm 0.05 & Maoli et al. $(2001)$ \\
0.81 & +0.14 & Hoekstra et al. (2002d) \\
0.97 & \pm 0.19 & Bacon et al. (2003) \\
0.72 & \pm 0.09 & Brown et al. (2003) \\
0.97 & \pm 0.35 & Hamana et al.(2003) \\
0.71 & +0.12 & Jarvis et al. (2003) \\
1.02 & \pm 0.16 & Rhodes et al. (2004) \\
0.83 & \pm 0.07 & Van Waerbeke et al. (2005) \\
\hline
\end{tabular}

\subsection{Potential systematics}

As the effects of weak lensing can be summarised by a scalar potential, only such distortion patterns can be caused by weak lensing which can be described by derivatives of a scalar potential. Possible patterns in the data resembling the derivatives of a vector potential thus signal the presence of distortions of other than lensing origin. Alluding to electrodynamics, where the electric field $\vec{E}$ is the gradient of a scalar potential and the magnetic field $\vec{B}$ is the curl of a vector potential, measured distortion patterns are decomposed into so-called $E$ and $B$ modes, of which only the $E$ modes can be caused by gravitational lensing. Significant $B$ modes in the data are interpreted as remainders of undetected or incompletely removed systematics.

More or less significant $B$ modes have been found in almost all weak-lensing surveys. Until recently, their origin was unclear. Schneider et al. (2002b) showed that source clustering could cause a $B$-mode contribution to the distortion, but not of sufficient strength on arc-minute scales to fully account for the observations. Hoekstra (2004) showed that incomplete correction for the anisotropies in the point-spread function of the imaging system could give rise to a substantial $B$ mode. Application of an improved model for the point-spread function to the Virmos-Descart weak-lensing survey caused the $B$ mode to 
disappear (Van Waerbeke et al.2005, see also Fig. 9). It thus appears that the $B$-mode problem, which was discussed at length in the literature, was due to insufficient correction for the distortions imprinted by the imaging system.

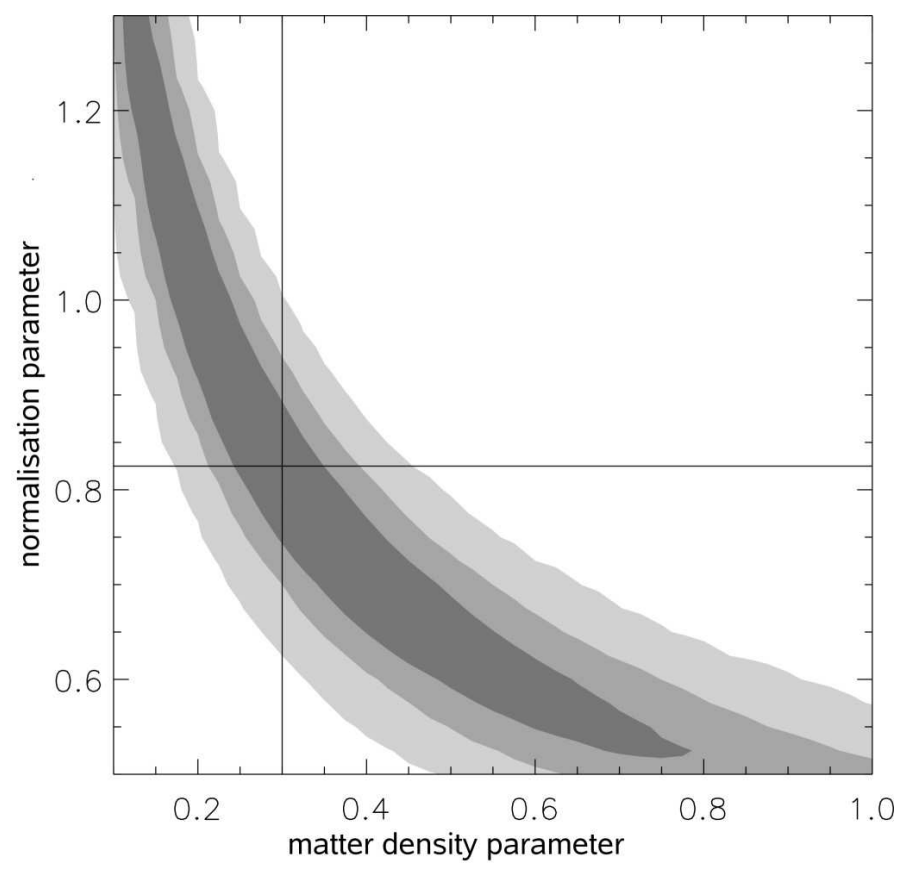

FIG. 9.-Likelihood contours (at $68 \%, 95 \%$, and $99.9 \%$ confidence) obtained from analyses of the cosmological weak shear in the plane spanned by the matter density parameter $\Omega_{\mathrm{m}}$ and the normalisation parameter $\sigma_{8}$. The solid lines indicate the value of $\sigma_{8}$ corresponding to $\Omega_{\mathrm{m}}=0.3$. (adapted from Van Waerbeke et al. 2005)

Analyses of cosmic-shear measurements assume that intrinsic galaxy ellipticities are uncorrelated such that they average to zero when several images are combined. However, galaxies being physically close to each other are also expected to have their shapes aligned, e.g. by the tidal field of the large-scale matter distribution into which they are embedded. The potential effect of intrinsic rather than lensing-induced galaxy alignments depends obviously on the depth of the survey. Deep surveys project galaxy images along light paths which are substantially longer than any large-scale structure correlation scale and thus suppress any spurious signal due to intrinsinc alignments of physically neighbouring galaxies. In shallow surveys, however, intrinsic source alignments may substantially contaminate any weak-shear signal (Croft \& Metzler 2000; Heavens et al. 2000 Catelan et al. 2001; Crittenden et al. 2001). Measurements of intrinsic alignments were found to agree well with these predictions (Lee \& Pen 2002, Brown et al. 2002). Recent numerical (Jing 2002) and analytic studies (Lee 2004) claim strong intrinsic alignments of galaxy halos.

Possibilities for removing the signal contamination due to intrinsic alignments were discussed extensively. They advocate using photometric redshifts to remove physically close pairs of source galaxies from the analysis (King \& Schneider 2002. Heymans \& Heavens 2003). An application of this technique to the multi-colour Combo-17 survey (Heymans et al. 2004) showed intrinsic alignments near the lower end of the theoretical predictions and slightly lowered the value of $\sigma_{8}$ previously derived from these data (Brown et al. 2003) to $0.67 \pm 0.1$ for $\Omega_{0}=0.3$. Hirata \& Seljak (2004) point out that foreground galaxies are aligned with the large-scale structures lensing background galaxies, thus giving rise to a higher-order alignment between galaxies at different redshifts.

\subsection{Perspectives}

For several years now, cosmological parameters are obtained with high accuracy mainly from combined analyses of CMB data, large-scale galaxy surveys and the dimming of type-Ia supernovae with redshift. What is the role of weak gravitational lensing in this context? It should be emphasised that parameter constraints from the CMB alone suffer from degeneracies which can only be broken using additional information. Measuring directly the dark-matter density and the normalisation of its fluctuations, gravitational lensing adds constraints which substantially narrow the parameter ranges allowed by the $\mathrm{CMB}$, as $\mathrm{Hu} \&$ Tegmark (1999) pointed out. Contaldi et al. (2003) combined weak-lensing data from the Red-sequence Cluster Survey with CMB data from the WMAP satellite to find $\sigma_{8}=0.89 \pm 0.05, \Omega_{0}=0.30 \pm 0.03$ and a Hubble constant of $H_{0}=(70 \pm 3) \mathrm{km} \mathrm{s}^{-1} \mathrm{Mpc}^{-1}$. Tereno et al. (2005) predicted the accuracy of joint cosmological parameter estimates using CMB data in combination with the weak-lensing constraints from the CFHT Legacy Survey.

The exploitation of higher than second-order statistics will become increasingly important for breaking degeneracies in the weak-lensing parameter estimates and the investigation of non-Gaussianity developing due to non-linear structure growth (Zhang et al. 2003; Takada \& Jain 2003, Dolney et al. 2004). Much valuable information must also be contained in the morphology of the two-dimensional weak-lensing pattern.

Among the most exciting perspectives of weak lensing is its potential to study the three-dimensional distribution of dark structures. Although lensing observables measure the twodimensional, projected density distribution, selecting sources at different distances allows structures along the line-of-sight to be resolved. Sufficiently accurate information on the distance to the source galaxies is provided by photometric redshifts. Simon et al. (2004) show that the accuracy of cosmic-shear parameter estimates can be improved by a factor of $5 \ldots 10$ by splitting the source galaxies into only four redshift bins. Heavens (2003) developed an elegant formalism for extracting three-dimensional information from weak-lensing data (see also Pen 2004a), and Taylor et al. (2004) recovered the three-dimensional matter distribution in the field of the Combo-17 survey, using photometric redshifts as distance indicator for the source galaxies.

The possibility to extract three-dimensional information from weak-lensing data opens a way for studying the growth of cosmic structures along the line-of-sight from the distant and past Universe. This, in turn, is sensitive to the change of the darkenergy density with time. Thus, sufficiently accurate and widefield weak-lensing surveys will allow the dark energy to be constrained (Huterer 2002). The sensitivity of such experiments is very promising (Munshi \& Wang 2003, Benabed \& van Waerbeke 2004), in particular if higher-order statistics are considered. This is one of the strongest motivations for a weak-lensing survey from space (Massey et al.|2004, Refregier et al.|2004).

Planned wide-area surveys of the sky in radio wavebands are expected to yield information on both cosmology and the distribution of neutral hydrogen during reionisation (Pen 2004b). Weak lensing in the data of the FIRST radio survey undertaken at $20 \mathrm{~cm}$ wavelength was recently detected by (Chang et al. 2004).

\subsection{Cosmic magnification}

\subsubsection{Magnification bias}

Besides distorting the images of distant galaxies, large-scale structures also magnify background sources. To linear order, the power spectrum of cosmic magnification is simply four times the 
power spectrum of the cosmic shear, i.e. both contain the same amount of cosmological information. Cosmic shear, however, is much more easily measurable than cosmic magnification because it can reasonably be assumed that the ellipticities of distant galaxies average to zero. The intrinsic flux of any given source being unknown, cosmic magnification is much harder to identify.

Currently the most promising (and perhaps the only) method for detecting cosmic magnification is the magnification bias. If a population of distant sources, e.g. quasars, is observed within a solid angle $\delta \Omega$ on the sky where the magnification is $\mu$, fainter sources become visible there, but their number density is reduced because the solid angle is stretched by the magnification. The net effect depends on how many more sources the magnification lifts above the flux threshold of the observation. If the number-count function of the sources is sufficiently steep, the dilution is outweighed and the magnification causes more sources to be visible, while sources with flat number counts are diluted and appear less numerous than without the magnification.

The spatial galaxy distribution follows the dark-matter structures which act as lenses on background sources. Bright quasars, for example, have a steep number-count function and thus appear more numerous behind magnifying dark-matter overdensities. The correlation of foreground galaxies with the lensing matter then leads to a positive cross-correlation on angular scales of arc minutes and larger between distant QSOs and foreground galaxies which are physically separated by cosmological distances.

The cross-correlation function between background sources and foreground galaxies was derived in linear approximation by Bartelmann (1995b) and Dolag \& Bartelmann (1997); see also Guimarães et al. (2001). However, magnification is non-linear in shear and convergence. Computing the theoretical expectation of the cosmic magnification accurately is thus considerably more complicated than for the cosmic shear. Ménard et al. (2003b) derived second-order corrections to the linear cosmicmagnification statistics and found that the linear approximation was systematically low by $20 \% \ldots 30 \%$, which was confirmed by Takada \& Hamana (2003) in a fully non-linear treatment based on the halo model of the dark-matter distribution. The accuracy of analytic calculations can be calibrated using numerical simulations of light propagation through large-scale structures (e.g. Barber \& Taylor 2003, Ménard et al. 2003b). Sufficiently accurate analytic and numerical calculations of cosmic magnification yield typical magnifications of $\lesssim 10 \%$ for point sources at redhifts $z_{\mathrm{s}} \simeq 1$. While most emphasis was put on cross-correlations between QSOs and galaxies, cosmic magnification may also induce detectable cross-correlations between background and foreground galaxies (Moessner \& Jain 1998 Moessner et al. 1998). Jain (2002) discussed survey strategies for detecting cosmic magnification.

\subsubsection{Observations}

Bartelmann \& Schneider (1993a) confirmed that the QSOgalaxy correlations detected by Seldner \& Peebles (1979) and Fugmann (1990) can indeed be explained in terms of gravitational lensing by large-scale structures. Subsequent more detailed correlation studies confirmed that the correlations showed the significance variations expected from the lensing hypothesis (Bartelmann \& Schneider 1993b), and revealed cross-correlations of distant QSOs with infrared (Bartelmann \& Schneider 1994, Bartsch et al. 1997) and X-ray emission (Bartelmann et al. 1994). While the existence of QSOgalaxy cross-correlations was thus firmly established, their amplitude and angular scale was typically found to be substantially too high (Williams \& Irwin 1998; Norman \& Williams 2000, Benítez et al. 2001, Gaztañaga 2003), although some analyses obtained the theoretically expected results (Rodrigues-Williams \& Hogan 1994, Norman \& Impey 2001). The observational situation was thus utterly confused. Fort et al. (1996) found evidence for cosmic shear in the vicinity of five distant QSOs. Dust absorption in foreground galaxies may be responsible for similar anti-correlations as expected from weak lensing of faint background QSOs (e.g. Croom 2001), and selection effects may cause correlations as well as anti-correlations (e.g. Ferreras et al. 1997). The detailed relation between dark-matter halos and their galaxy content adds further uncertainty (Jain et al. 2003).

Due to the weakness of the signal and the possibility of confusing it with other effects, large multi-colour surveys such as the SDSS were expected to be necessary for an unambiguous detection of cosmic magnification and its exploitation in terms of cosmological parameters (Benítez \& Sanz 1999, Ménard \& Bartelmann 2002). The degeneracy between the density and bias parameters $\Omega_{0}$ and $b$ can be broken determining the three-point cross-correlation between QSOs and galaxy pairs (Ménard et al. 2003a). Clear and unambiguous evidence for cosmic magnification was finally detected by Scranton et al. (2005) who measured the cross-correlation between a colour-selected sample of $\simeq 200,000$ distant QSOs and foreground galaxies in $\simeq 3,800$ square degrees of the SDSS data and found a signal significant at the $8-\sigma$ level and in complete agreement with theoretical expectations.

A potentially very promising manifestation of cosmic magnification was recently discussed by Ménard \& Péroux (2003). Distant QSOs magnified by intervening matter are more likely to show absorption features in their spectra due to the gas associated with the lensing structures. Using the QSO sample from the $2 \mathrm{dF}$ survey, Ménard \& Péroux (2003) demonstrated evidence for lensing by MgII and FeII absorbers along the lines-of-sight to the QSOs.

\section{SUMMARY}

Many are the applications of gravitational lensing to cosmology, and the results are numerous, as the preceding discussion has shown. A review like this must be based on a subjective selection which is necessarily biased to some degree. Within these limitations, I summarise the results as follows:

- Microlensing experiments in the Galaxy have shown that, although massive compact objects exist in its halo, they insufficient to make up all the dark matter in the Galactic halo. These studies are now being extended towards the Andromeda galaxy, M 31 (Sect. 3.1).

- Central density profiles of lensing galaxies are approximately isothermal and thus more concentrated than CDM predicts. This indicates that galaxy density profiles have been steepened by baryonic physics (Sect. 3.2.1).

- The isothermal density profiles and the characteristic velocity dispersions of galaxies are confirmed by weak galaxygalaxy lensing, which finds very large halo sizes with radii of $\gtrsim 200 h^{-1} \mathrm{kpc}$. Halos of cluster galaxies seem to be smaller, as expected (Sect. 3.2.5).

- The biasing of galaxies relative to the dark-matter distribution is found by galaxy-galaxy lensing to be almost scaleindependent, or gently increasing with scale (Sect. 3.2.5).

- Galaxies have to be structured in order to explain multipleimage geometries and the high fraction of quadruple com- 
pared to double images. Anomalous flux ratios of quadruple images seem to be best explained by lensing, but it is yet unclear whether they are caused by the galactic subhalos expected in CDM (Sect. 3.2.3.

- Measured time delays between multiple images lead to an interesting and as yet unresolved conflict between the lensing mass distribution and the Hubble constant: Isothermal profiles yield Hubble constants which are substantially too low, and lens models giving compatible Hubble constants have too steep mass profiles (Sect.3.2.2).

- The statistics of distant sources multiply imaged by galaxies is sensitive to the cosmological parameters. Recent applications of this method showed agreement with a lowdensity universe with cosmological constant (Sect. 3.2.4).

- Galaxy clusters have to be asymmetric, and they must be dominated by dark matter which is more broadly distributed than the cluster light. Cores in the dark-matter distribution must be small or absent (Sect.4.1.1.4.1.3.

- Frequent and substantial discrepancies between lensing and $\mathrm{X}$-ray mass determinations are most likely signalling violent dynamical activity in clusters (Sect.4.1.2, 4.3.2.

- It remains unclear whether galaxy clusters in the "concordance", low-density spatially-flat cosmological models can explain the observed abundance of gravitational arcs. Clusters need to be highly substructured and asymmetric, and their dynamics may temporarily boost their strong-lensing efficiency (Sect.4.1.4).

- Although cluster density profiles inferred from strong and weak lensing do typically not contradict expectations from CDM, isothermal density profiles are not ruled out. Claims of flat central profiles are not supported by reasonably asymmetric models (Sect. 4.1.3, 4.3.3.

- Typical mass-to-light ratios derived from weak cluster lensing range around $\simeq 200$ in solar units, but very high values have been found. While this may indicate a separation of gas from dark matter in cluster mergers, the possible existence of dark clusters is intriguing (Sect.4.3.2, 4.3.4).

- Surprisingly massive and compact clusters which are significant weak and powerful strong lenses exist at redshifts $z \simeq 0.8$ and above (Sect. 4.1.4, 4.3.3.

- Cosmic shear, i.e. the distortion of background-galaxy images due to weak gravitational lensing by large-scale structures, has been detected and found to be in remarkable agreement with theoretical expectations. It has allowed constraints to be placed on the matter-density parameter and the amplitude of the dark-matter fluctuations (Sect. 5.1).

- Systematic effects such as possible intrinsic alignments of source galaxies and the so-called $B$ mode which is evidently not of lensing origin now seem to be well under control (Sect. 5.2).

- Joint analyses of weak lensing and CMB data allow parameter degeneracies in both types of experiment to be lifted. When combined with photometric redshifts of source galaxies, three-dimensional reconstructions of the large-scale matter distribution become possible. This will also allow the dark energy to be constrained (Sect. 5.3).
- Cosmic magnification, which is more complicated to measure than cosmic shear, can be quantified by the magnification bias. It has been detected, and most recent measurements are also in excellent agreement with theoretical expectations (Sect. 5.4.

\section{REFERENCES}

Alcock, C., Allsman, R. A., Alves, D. R., et al. 2000, ApJ, 542, 281

Allen, S. W. 1998, MNRAS, 296, 392

Arabadjis, J. S., Bautz, M. W., \& Garmire, G. P. 2002, ApJ, 572, 66

Athreya, R. M., Mellier, Y., van Waerbeke, L., et al. 2002, A\&A, 384, 743

Aubourg, É., Palanque-Delabrouille, N., Salati, P., Spiro, M., \& Taillet, R. 1999, A\&A, 347, 850

Bacon, D. J., Massey, R. J., Refregier, A. R., \& Ellis, R. S. 2003, MNRAS, 344 673

Bacon, D. J., Refregier, A. R., \& Ellis, R. S. 2000, MNRAS, 318, 625

Barber, A. J. \& Taylor, A. N. 2003, MNRAS, 344, 789

Bartelmann, M. 1995a, A\&A, 299, 11

Bartelmann, M. 1995b, A\&A, 298, 661

Bartelmann, M. 1996, A\&A, 313, 697

Bartelmann, M., Huss, A., Colberg, J. M., Jenkins, A., \& Pearce, F. R. 1998, A\&A, 330, 1

Bartelmann, M., King, L. J., \& Schneider, P. 2001, A\&A, 378, 361

Bartelmann, M. \& Meneghetti, M. 2004, A\&A, 418, 413

Bartelmann, M., Meneghetti, M., Perrotta, F., Baccigalupi, C., \& Moscardini, L. 2003, A\&A, 409, 449

Bartelmann, M., Narayan, R., Seitz, S., \& Schneider, P. 1996, ApJL, 464, L115+

Bartelmann, M., Perrotta, F., \& Baccigalupi, C. 2002, A\&A, 396, 21

Bartelmann, M. \& Schneider, P. 1992, A\&A, 259, 413

Bartelmann, M. \& Schneider, P. 1993a, A\&A, 268, 1

Bartelmann, M. \& Schneider, P. 1993b, A\&A, 271, 42

Bartelmann, M. \& Schneider, P. 1994, A\&A, 284, 1

Bartelmann, M. \& Schneider, P. 2001, Phys. Rep., 340, 291

Bartelmann, M., Schneider, P., \& Hasinger, G. 1994, A\&A, 290, 399

Bartelmann, M. \& Steinmetz, M. 1996, MNRAS, 283, 431

Bartelmann, M., Steinmetz, M., \& Weiss, A. 1995, A\&A, 297, 1

Bartelmann, M. \& Weiss, A. 1994, A\&A, 287, 1

Bartsch, A., Schneider, P., \& Bartelmann, M. 1997, A\&A, 319, 375

Benabed, K. \& van Waerbeke, L. 2004, Phys. Rev. D, 70, 123515

Benítez, N. \& Sanz, J. L. 1999, ApJL, 525, L1

Benítez, N., Sanz, J. L., \& Martínez-González, E. 2001, MNRAS, 320, 241

Bergmann, A. G., Petrosian, V., \& Lynds, R. 1990, ApJ, 350, 23

Bernardeau, F., Mellier, Y., \& van Waerbeke, L. 2002, A\&A, 389, L28

Bernardeau, F., van Waerbeke, L., \& Mellier, Y. 1997, A\&A, 322, 1

Blandford, R. D., Saust, A. B., Brainerd, T. G., \& Villumsen, J. V. 1991, MNRAS, 251, 600

Bradač, M., Schneider, P., Lombardi, M., et al. 2004, A\&A, 423, 797

Bradač, M., Schneider, P. Steinmetz, M., et al. 2002, A\&A, 388, 373

Brainerd, T. G., Blandford, R. D., \& Smail, I. 1996, ApJ, 466, 623

Brown, M. L., Taylor, A. N., Bacon, D. J., et al. 2003, MNRAS, 341, 100

Brown, M. L., Taylor, A. N., Hambly, N. C., \& Dye, S. 2002, MNRAS, 333, 501

Casoli, F., Encrenaz, P., Fort, B., Boisse, P., \& Mellier, Y. 1996, A\&A, 306, L41+

Catelan, P., Kamionkowski, M., \& Blandford, R. D. 2001, MNRAS, 320, L7

Chae, K.-H., Biggs, A. D., Blandford, R. D., et al. 2002, Physical Review Letters, 89,151301

Chang, T., Refregier, A., \& Helfand, D. J. 2004, ApJ, 617, 794

Chen, J., Kravtsov, A. V., \& Keeton, C. R. 2003, ApJ, 592, 24

Chiba, M. 2002, ApJ, 565, 17

Chiba, M. \& Yoshii, Y. 1999, ApJ, 510, 42

Clowe, D., De Lucia, G., \& King, L. 2004, MNRAS, 350, 1038

Clowe, D., Luppino, G. A., Kaiser, N., \& Gioia, I. M. 2000, ApJ, 539, 540

Clowe, D., Luppino, G. A., Kaiser, N., Henry, J. P., \& Gioia, I. M. 1998, ApJL, 497, L61+

Clowe, D. \& Schneider, P. 2001, A\&A, 379, 384

Clowe, D. \& Schneider, P. 2002, A\&A, 395, 385

Cohn, J. D. \& Kochanek, C. S. 2004, ApJ, 608, 25

Contaldi, C. R., Hoekstra, H., \& Lewis, A. 2003, Physical Review Letters, 90, 221303

Cooray, A. \& Hu, W. 2001, ApJ, 554, 56

Cooray, A. R. 1999, A\&A, 341, 653

Crittenden, R. G., Natarajan, P., Pen, U., \& Theuns, T. 2001, ApJ, 559, 552

Croft, R. A. C. \& Metzler, C. A. 2000, ApJ, 545, 561

Croom, S. M. 2001, Publications of the Astronomical Society of Australia, 18, 169

Cypriano, E. S., Sodré, L. J., Kneib, J., \& Campusano, L. E. 2004, ApJ, 613, 95

Dahle, H., Kaiser, N., Irgens, R. J., Lilje, P. B., \& Maddox, S. J. 2002, ApJS, 139,313

Dahle, H., Pedersen, K., Lilje, P. B., Maddox, S. J., \& Kaiser, N. 2003, ApJ, 591,662

Dalal, N., Holder, G., \& Hennawi, J. F. 2004, ApJ, 609, 50

Dalal, N. \& Kochanek, C. S. 2002, ApJ, 572, 25

de Jong, J. T. A., Kuijken, K., Crotts, A. P. S., et al. 2004, A\&A, 417, 461

dell'Antonio, I. P. \& Tyson, J. A. 1996, ApJL, 473, L17+

Dolag, K. \& Bartelmann, M. 1997, MNRAS, 291, 446 
Dolney, D., Jain, B., \& Takada, M. 2004, MNRAS, 352, 1019

Eddington, A. S. 1919, The Observatory, 42, 119

Erben, T., Miralles, J. M., Clowe, D., et al. 2003, A\&A, 410, 45

Erben, T., Van Waerbeke, L., Bertin, E., Mellier, Y., \& Schneider, P. 2001, A\&A 366,717

Erben, T., van Waerbeke, L., Mellier, Y., et al. 2000, A\&A, 355, 23

Evans, N. W. \& Kerins, E. 2000, ApJ, 529, 917

Evans, N. W. \& Witt, H. J. 2003, MNRAS, 345, 1351

Fahlman, G., Kaiser, N., Squires, G., \& Woods, D. 1994, ApJ, 437, 56

Falco, E. E., Kochanek, C. S., \& Munoz, J. A. 1998, ApJ, 494, 47

Fassnacht, C. D., Xanthopoulos, E., Koopmans, L. V. E., \& Rusin, D. 2002, ApJ, 581,823

Ferreras, I., Benitez, N., \& Martinez-Gonzalez, E. 1997, AJ, 114, 1728

Fischer, P. 1999, AJ, 117, 2024

Fischer, P., McKay, T. A., Sheldon, E., et al. 2000, AJ, 120, 1198

Flores, R. A., Maller, A. H., \& Primack, J. R. 2000, ApJ, 535, 555

Fort, B., Le Fevre, O., Hammer, F., \& Cailloux, M. 1992, ApJL, 399, L125

Fort, B., Mellier, Y., Dantel-Fort, M., Bonnet, H., \& Kneib, J.-P. 1996, A\&A, 310,705

Frye, B. \& Broadhurst, T. 1998, ApJL, 499, L115+

Fugmann, W. 1990, A\&A, 240, 11

Gautret, L., Fort, B., \& Mellier, Y. 2000, A\&A, 353, 10

Gavazzi, R., Fort, B., Mellier, Y., Pelló, R., \& Dantel-Fort, M. 2003, A\&A, 403,

Gavazzi, R., Mellier, Y., Fort, B., Cuillandre, J.-C., \& Dantel-Fort, M. 2004 A\&A, 422, 407

Gaztañaga, E. 2003, ApJ, 589, 82

Geiger, B. \& Schneider, P. 1998, MNRAS, 295, 497

Geiger, B. \& Schneider, P. 1999, MNRAS, 302, 118

Gioia, I. M., Henry, J. P., Mullis, C. R., Ebeling, H., \& Wolter, A. 1999, AJ, 117 2608

Gladders, M. D., Hoekstra, H., Yee, H. K. C., Hall, P. B., \& Barrientos, L. F 2003, ApJ, 593, 48

Golse, G. \& Kneib, J.-P. 2002, A\&A, 390, 821

Gould, A. 1993, ApJ, 404, 45

Gould, A. 1995, ApJ, 441, 77

Gray, M. E., Ellis, R. S., Lewis, J. R., McMahon, R. G., \& Firth, A. E. 2001 , MNRAS, 325, 111

Gray, M. E., Taylor, A. N., Meisenheimer, K., et al. 2002, ApJ, 568, 141

Grossman, S. A. \& Narayan, R. 1988, ApJL, 324, L37

Guimarães, A. C. C., van De Bruck, C., \& Brandenberger, R. H. 2001, MNRAS, 325,278

Guzik, J. \& Seljak, U. 2002, MNRAS, 335, 311

Gyuk, G., Dalal, N., \& Griest, K. 2000, ApJ, 535, 90

Hamana, T. \& Mellier, Y. 2001, MNRAS, 327, 169

Hamana, T., Miyazaki, S., Shimasaku, K., et al. 2003, ApJ, 597, 98

Hammer, F. \& Rigaut, F. 1989, A\&A, 226, 45

Hammer, F., Rigaut, F., Le Fevre, O., Jones, J., \& Soucail, G. 1989, A\&A, 208 L7

Hattori, M., Matuzawa, H., Morikawa, K., et al. 1998, ApJ, 503, 593

Hattori, M. Watanabe, K, \& Yamashita, K. 1997, A\&A, 319, 764

Heavens, A. 2003, MNRAS, 343, 1327

Heavens, A., Refregier, A., \& Heymans, C. 2000, MNRAS, 319, 649

Heymans, C., Brown, M., Heavens, A., et al. 2004, MNRAS, 347, 895

Heymans, C. \& Heavens, A. 2003, MNRAS, 339, 711

Hirata, C. M., Mandelbaum, R., Seljak, U., et al. 2004, MNRAS, 353, 529

Hirata, C. M. \& Seljak, U. 2004, Phys. Rev. D, 70, 063526

Hoekstra, H. 2003, MNRAS, 339, 1155

Hoekstra, H. 2004, MNRAS, 347, 1337

Hoekstra, H., Franx, M., \& Kuijken, K. 2000, ApJ, 532, 88

Hoekstra, H., Franx, M., Kuijken, K., \& van Dokkum, P. G. 2002a, MNRAS, 333,911

Hoekstra, H., van Waerbeke, L., Gladders, M. D., Mellier, Y., \& Yee, H. K. C. 2002b, ApJ, 577, 604

Hoekstra, H., Yee, H. K. C., \& Gladders, M. D. 2001, ApJL, 558, L11

Hoekstra, H., Yee, H. K. C., \& Gladders, M. D. 2002c, New Astronomy Review, 46, 767

Hoekstra, H., Yee, H. K. C., \& Gladders, M. D. 2004, ApJ, 606, 67

Hoekstra, H., Yee, H. K. C., Gladders, M. D., et al. 2002d, ApJ, 572, 55

Hogg, D. W. \& Blandford, R. D. 1994, MNRAS, 268, 889

Holden, B. P., Stanford, S. A., Squires, G. K., et al. 2002, AJ, 124, 33

Hu, W. \& Tegmark, M. 1999, ApJL, 514, L65

Hu, W. \& White, M. 2001, ApJ, 554, 67

Hubble, E. 1929, Proceedings of the National Academy of Science, 15, 168

Hubble, E. \& Humason, M. L. 1931, ApJ, 74, 43

Huterer, D. 2002, Phys. Rev. D, 65, 063001

Inada, N., Oguri, M., Pindor, B., et al. 2003, Nat, 426, 810

Irgens, R. J.,Lilje, P. B., Dahle, H., \& Maddox, S. J. 2002, ApJ, 579, 227

Jain, B. 2002, ApJL, 580, L3

Jain, B., Scranton, R., \& Sheth, R. K. 2003, MNRAS, 345, 62

Jain, B. \& Seljak, U. 1997, ApJ, 484, 560

Jain, B., Seljak, U., \& White, S. 2000, ApJ, 530, 547

Jarvis, M., Bernstein, G. M., Fischer, P., et al. 2003, AJ, 125, 1014

Jee, M. J., White, R. L., Benítez, N., et al. 2005, ApJ, 618, 46

Jenkins, A., Frenk, C. S., White, S. D. M., et al. 2001, MNRAS, 321, 372

Jetzer, P., Mancini, L., \& Scarpetta, G. 2002, A\&A, 393, 129

Jia, S. M., Chen, Y., Lu, F. J., Chen, L., \& Xiang, F. 2004, A\&A, 423, 65

Jing, Y. P. 2002, MNRAS, 335, L89

Kaiser, N. 1992, ApJ, 388, 272

Kaiser, N. 1998, ApJ, 498, 26
Kaiser, N. \& Squires, G. 1993, ApJ, 404, 44

Kaiser, N., Squires, G., \& Broadhurst, T. 1995, ApJ, 449, 460

Kassiola, A., Kovner, I., \& Blandford, R. D. 1992, ApJ, 396, 10

Kaufmann, R. \& Straumann, N. 2000, Annalen der Physik, 9, 384

Keeton, C. R. 2001, ApJ, 561, 46

Keeton, C. R. 2002, ApJL, 575, L

Keeton, C. R., Kochanek, C. S., \& Seljak, U. 1997, ApJ, 482, 604

Keeton, C. R. \& Zabludoff, A. I. 2004, ApJ, 612, 660

King, L. \& Schneider, P. 2002, A\&A, 396, 411

King, L. J., Schneider, P., \& Springel, V. 2001, A\&A, 378, 748

Kneib, J., Ellis, R. S., Santos, M. R., \& Richard, J. 2004, ApJ, 607, 697

Kneib, J., Hudelot, P., Ellis, R. S., et al. 2003, ApJ, 598, 804

Kneib, J.-P., Ellis, R. S., Smail, I., Couch, W. J., \& Sharples, R. M. 1996, ApJ, 471,643

Kochanek, C. S. 1995 , ApJ 453,545

Kochanek, C. S. 1996, ApJ, 466, 638

Kochanek, C. S. 2003, ApJ, 583, 49

Kochanek, C. S. \& Dalal, N. 2004, ApJ, 610, 69

Koopmans, L. V. E., Treu, T., Fassnacht, C. D., Blandford, R. D., \& Surpi, G. 2003, ApJ, 599, 70

Kormann, R., Schneider, P., \& Bartelmann, M. 1994, A\&A, 286, 357

Kovner, I. 1989, ApJ, 337, 621

Kruse, G. \& Schneider, P. 1999, MNRAS, 302, 821

Lasserre, T., Afonso, C., Albert, J. N., et al. 2000, A\&A, 355, L39

Le Fevre, O., Hammer, F., Angonin, M. C., Gioia, I. M., \& Luppino, G. A. 1994 ApJL, 422, L5

Lee, J. 2004, ApJL, 614, L1

Lee, J. \& Pen, U. 2002, ApJL, 567, L111

Lewis, A. D., Ellingson, E., Morris, S. L., \& Carlberg, R. G. 1999, ApJ, 517, 587

Link, R. \& Pierce, M. J. 1998, ApJ, 502, 63

Luppino, G. A., Gioia, I. M., Hammer, F., Le Fèvre, O., \& Annis, J. A. 1999, A\&AS, 136, 117

Luppino, G. A. \& Kaiser, N. 1997, ApJ, 475, 20

Lynds, R. \& Petrosian, V. 1989, ApJ, 336, 1

Ménard, B. \& Bartelmann, M. 2002, A\&A, 386, 784

Ménard, B., Bartelmann, M., \& Mellier, Y. 2003a, A\&A, 409, 411

Ménard, B., Hamana, T., Bartelmann, M., \& Yoshida, N. 2003b, A\&A, 403, 817

Ménard, B. \& Péroux, C. 2003, A\&A, 410, 33

Möller, O., Hewett, P., \& Blain, A. W. 2003, MNRAS, 345, 1

Machacek, M. E., Bautz, M. W., Canizares, C., \& Garmire, G. P. 2002, ApJ 567,188

Makino, N. \& Asano, K. 1999, ApJ, 512, 9

Mancini, L., Calchi Novati, S., Jetzer, P., \& Scarpetta, G. 2004, A\&A, 427, 61

Mao, S., Jing, Y., Ostriker, J. P., \& Weller, J. 2004, ApJL, 604, L5

Mao, S. \& Schneider, P. 1998, MNRAS, 295, 587

Maoli, R., Van Waerbeke, L., Mellier, Y., et al. 2001, A\&A, 368, 766

Margoniner, V. E., Lubin, L. M., Wittman, D. M., \& Squires, G. K. 2005, AJ, 129,20

Markevitch, M., Gonzalez, A. H., Clowe, D., et al. 2004, ApJ, 606, 819

Marshall, P. J., Hobson, M. P., Gull, S. F., \& Bridle, S. L. 2002, MNRAS, 335 , 1037

Massey, R., Rhodes, J., Refregier, A., et al. 2004, AJ, 127, 3089

Mathez, G., Fort, B., Mellier, Y., Picat, J.-P., \& Soucail, G. 1992, A\&A, 256 343

Mellier, Y. 1999, ARA\&A, 37, 127

Mellier, Y., Fort, B., \& Kneib, J. 1993, ApJ, 407, 33

Mellier, Y. \& van Waerbeke, L. 2002, Classical and Quantum Gravity, 19, 3505

Meneghetti, M., Bartelmann, M., \& Moscardini, L. 2003a, MNRAS, 346, 67

Meneghetti, M., Bartelmann, M., \& Moscardini, L. 2003b, MNRAS, 340, 105

Meneghetti, M., Bolzonella, M., Bartelmann, M., Moscardini, L., \& Tormen, G. 2000, MNRAS, 314, 338

Meneghetti, M., Yoshida, N., Bartelmann, M., et al. 2001, MNRAS, 325, 435

Metcalf, R. B. 2002, ApJ, 580, 696

Metcalf, R. B. \& Zhao, H. 2002, ApJL, 567, L5

Metzler, C. A., White, M., Norman, M., \& Loken, C. 1999, ApJL, 520, L9

Miralda-Escudé, J. 1991, ApJ, 380,

Miralda-Escudé, J. 1993, ApJ, 403, 497

Miralda-Escudé, J. 1995, ApJ, 438, 514

Miralda-Escudé, J. 2002, ApJ, 564, 60

Miralda-Escudé, J. \& Babul, A. 1995, ApJ, 449, 18

Miralles, J.-M., Erben, T., Hämmerle, H., et al. 2002, A\&A, 388, 68

Miyazaki, S., Hamana, T., Shimasaku, K, et al. 2002, ApJL, 580, L97

Moessner, R. \& Jain, B. 1998, MNRAS, 294, L18

Moessner, R., Jain, B., \& Villumsen, J. V. 1998, MNRAS, 294, 291

Molikawa, K. \& Hattori, M. 2001, ApJ, 559, 544

Molikawa, K., Hattori, M., Kneib, J., \& Yamashita, K. 1999, A\&A, 351, 413

Mould, J., Blandford, R., Villumsen, J., et al. 1994, MNRAS, 271, 31

Munshi, D. \& Wang, Y. 2003, ApJ, 583, 566

Narayan, R. \& White, S. D. M. 1988, MNRAS, 231, 97P

Natarajan, P. \& Kneib, J. 1997, MNRAS, 287, 833

Natarajan, P., Kneib, J., \& Smail, I. 2002a, ApJL, 580, L1

Natarajan, P., Loeb, A., Kneib, J., \& Smail, I. 2002b, ApJL, 580, L17

Navarro, J. F., Frenk, C. S., \& White, S. D. M. 1996, ApJ, 462, 563

Navarro, J. F., Frenk, C. S., \& White, S. D. M. 1997, ApJ, 490, 493

Norman, D. J. \& Impey, C. D. 2001, AJ, 121, 2392

Norman, D. J. \& Williams, L. L. R. 2000, AJ, 119, 2060

Oguri, M. \& Keeton, C. R. 2004, ApJ, 610, 663

Oguri, M., Lee, J., \& Suto, Y. 2003, ApJ, 599, 7

Oguri, M., Taruya, A., \& Suto, Y. 2001, ApJ, 559, 572 
Ota, N., Mitsuda, K., \& Fukazawa, Y. 1998, ApJ, 495, 170

Ota, N., Pointecouteau, E., Hattori, M., \& Mitsuda, K. 2004, ApJ, 601, 120

Paczyński, B. 1986, ApJ, 304, 1

Paczyński, B. 1987, Nat, 325, 572

Paulin-Henriksson, S., Baillon, P., Bouquet, A., et al. 2003, A\&A, 405, 15

Pelló, R., Kneib, J. P., Le Borgne, J. F., et al. 1999, A\&A, 346, 359

Pelló, R., Sanahuja, B., Le Borgne, J., Soucail, G., \& Mellier, Y. 1991, ApJ, 366, 405

Pelló, R., Schaerer, D., Richard, J., Le Borgne, J.-F., \& Kneib, J.-P. 2004, A\&A, 416, L35

Pen, U. 2004a, MNRAS, 350, 1445

Pen, U. 2004b, New Astronomy, 9, 417

Phillips, P. M., Browne, I. W. A., Jackson, N. J., et al. 2001, MNRAS, 328, 1001

Pierre, M., Le Borgne, J. F., Soucail, G., \& Kneib, J. P. 1996, A\&A, 311, 413

Press, W. H. \& Schechter, P. 1974, ApJ, 187, 425

Quadri, R., Möller, O., \& Natarajan, P. 2003, ApJ, 597, 659

Reblinsky, K. \& Bartelmann, M. 1999, A\&A, 345, 1

Refregier, A. 2003, ARA\&A, 41, 645

Refregier, A., Massey, R., Rhodes, J., et al. 2004, AJ, 127, 3102

Rhodes, J., Refregier, A., Collins, N. R., et al. 2004, ApJ, 605, 29

Riffeser, A., Fliri, J., Bender, R., Seitz, S., \& Gössl, C. A. 2003, ApJL, 599, L17

Rodrigues-Williams, L. L. \& Hogan, C. J. 1994, AJ, 107, 451

Rusin, D., Kochanek, C. S., \& Keeton, C. R. 2003, ApJ, 595, 29

Rusin, D. \& Ma, C. 2001, ApJL, 549, L33

Sahu, K. C. 1994, PASP, 106, 942

Sand, D. J., Treu, T., Smith, G. P., \& Ellis, R. S. 2004, ApJ, 604, 88

Schechter, P. L. \& Wambsganss, J. 2002, ApJ, 580, 685

Schechter, P. L., Wambsganss, J., \& Lewis, G. F. 2004, ApJ, 613, 77

Schirmer, M., Erben, T., Schneider, P., Wolf, C., \& Meisenheimer, K. 2004 A\&A, 420, 75

Schneider, P. 1996, MNRAS, 283, 837

Schneider, P. \& Rix, H. 1997, ApJ, 474, 25

Schneider, P., van Waerbeke, L., Kilbinger, M., \& Mellier, Y. 2002a, A\&A, 396 1

Schneider, P., van Waerbeke, L., \& Mellier, Y. 2002b, A\&A, 389, 729

Schneider, P., van Waerbeke, L., Mellier, Y., et al. 1998, A\&A, 333, 767

Scranton, R., Ménard, B., Richards, G., et al. 2005, preprint

Seitz, C. \& Schneider, P. 1995, A\&A, 297, 287

Seitz, S. \& Schneider, P. 1996, A\&A, 305, 383

Seitz, S., Schneider, P., \& Bartelmann, M. 1998, A\&A, 337, 325

Seldner, M. \& Peebles, P. J. E. 1979, ApJ, 227, 30

Seljak, U. 2002, MNRAS, 334, 797

Sheldon, E. S., Annis, J., Böhringer, H., et al. 2001, ApJ, 554, 881

Sheldon, E. S., Johnston, D. E., Frieman, J. A., et al. 2004, AJ, 127, 2544

Sheth, R. K. \& Tormen, G. 2002, MNRAS, 329, 61

Simon, P., King, L. J., \& Schneider, P. 2004, A\&A, 417, 873

Smail, I., Hogg, D. W., Blandford, R., et al. 1995, MNRAS, 277, 1

Smith, D. R., Bernstein, G. M., Fischer, P., \& Jarvis, M. 2001, ApJ, 551, 643

Soucail, G., Fort, B., Mellier, Y., \& Picat, J. P. 1987, A\&A, 172, L14

Soucail, G., Kneib, J.-P., \& Golse, G. 2004, A\&A, 417, L33

Soucail, G., Mellier, Y., Fort, B., Mathez, G., \& Cailloux, M. 1988, A\&A, 191, L19

Takada, M. \& Hamana, T. 2003, MNRAS, 346, 949

Takada, M. \& Jain, B. 2003, MNRAS, 344, 857

Taylor, A. N., Bacon, D. J. Gray, M. E., et al. 2004, MNRAS, 353, 1176

Tereno, I., Doré, O., van Waerbeke, L., \& Mellier, Y. 2005, A\&A, 429, 383

Thompson, D., Pozzetti, L., Hasinger, G., et al. 2001, A\&A, 377, 778

Torri, E., Meneghetti, M., Bartelmann, M., et al. 2004, MNRAS, 349, 476

Treu, T. \& Koopmans, L. V. E. 2004, ApJ, 611, 739

Uglesich, R. R., Crotts, A. P. S., Baltz, E. A., et al. 2004, ApJ, 612, 877

Umetsu, K. \& Futamase, T. 2000, ApJL, 539, L5

Vale, C. \& White, M. 2003, ApJ, 592, 699

van Waerbeke, L., Bernardeau, F., \& Mellier, Y. 1999, A\&A, 342, 15

Van Waerbeke, L., Hamana, T., Scoccimarro, R., Colombi, S., \& Bernardeau, F 2001a, MNRAS, 322, 918

Van Waerbeke, L., Mellier, Y., Erben, T., et al. 2000, A\&A, 358, 30

Van Waerbeke, L., Mellier, Y, \& Hoekstra, H. 2005, A\&A, 429, 75

Van Waerbeke, L., Mellier, Y., Radovich, M., et al. 2001b, A\&A, 374, 757

Wambsganss, J., Bode, P., \& Ostriker, J. P. 2004, ApJL, 606, L93

Weinberg, D. H., Davé, R., Katz, N., \& Hernquist, L. 2004, ApJ, 601, 1

Weinberg, N. N. \& Kamionkowski, M. 2002, MNRAS, 337, 1269

Weinberg, N. N. \& Kamionkowski, M. 2003, MNRAS, 341, 251

White, M., van Waerbeke, L. \& Mackey, J. 2002, ApJ, 575, 640

Williams, L. L. R. \& Irwin, M. 1998, MNRAS, 298, 378

Williams, L. L. R., Navarro, J. F., \& Bartelmann, M. 1999, ApJ, 527, 535

Williams, L. L. R. \& Saha, P. 2004, AJ, 128, 2631

Wilson, G., Kaiser, N., Luppino, G. A., \& Cowie, L. L. 2001, ApJ, 555, 572

Winn, J. N., Rusin, D., \& Kochanek, C. S. 2004, Nat, 427, 613

Wittman, D., Tyson, J. A., Margoniner, V. E., Cohen, J. G., \& Dell' Antonio, I. P. 2001, ApJL, 557, L89

Wittman, D. M., Tyson, J. A., Kirkman, D., Dell'Antonio, I., \& Bernstein, G 2000, Nat, 405, 143

Wu, X. 1994a, ApJL, 436, L115

Wu, X. 1994 b, ApJ, 435, 66

Wu, X. 2000, MNRAS, 316, 299

Wu, X. \& Fang, L. 1996, ApJL, 467, L45+

Wu, X. \& Hammer, F. 1993, MNRAS, 262, 187

Yang, X. H., Mo, H. J., Kauffmann, G., \& Chu, Y. Q. 2003, MNRAS, 339, 387

Zaritsky, D. \& Gonzalez, A. H. 2003, ApJ, 584, 691

Zhang, T., Pen, U., Zhang, P., \& Dubinski, J. 2003, ApJ, 598, 818 